\title{
Risk-sharing and contagion in networks*
}

\author{
Antonio Cabrales \\ University College London
}

\author{
Piero Gottardi
}

European University Institute

\author{
Fernando Vega-Redondo \\ Università Bocconi \& IGIER
}

April 26, 2017

\begin{abstract}
We investigate the socially optimal design of financial networks that allow to tackle the trade-off between risk-sharing and contagion. We identify conditions on the shock distribution under which full integration or maximal segmentation is optimal. We also show that under different conditions the optimal network features varying strength of linkages to other firms or intermediate degrees of segmentation. In the latter case, the individual and social incentives to establish linkages are not necessarily aligned. When firms are heterogeneous in the distribution of risks they face, they should optimally form linkages only with firms facing risks of the same kind.
\end{abstract}

JEL Classification: D85, C72, G21.

Keywords: Financial networks, Contagion, Risk Sharing.

${ }^{*}$ We wish to thank Elena Carletti, Rama Cont, Maryam Farboodi, Sanjeev Goyal, Matt Jackson, Lasse Loepfe, Anxo Sánchez and Branko Urosevic for helpful comments and discussions. We are also grateful to the Co-Editor, Itay Goldstein, and two anonymous referees for very useful suggestions. An earlier version of this paper was presented at the Cambridge Workshop on Financial Networks, 2010, the SAET Conference in Faro, 2011, the Networks Conference at Ankara, 2012, the 2014 Wharton Conference on Liquidity and Financial Crises and various seminars; we are grateful to participants for their comments. We acknowledge financial support from the EUI Research Council, MIUR Prin prot. 2010NE9L9Z-004 and the Spanish Ministry of the Economy and Competitiveness under grants ECO2012-34581 and RESINEE. Send correspondence to Piero Gottardi, European University Institute, Department of Economics, Via delle Fontanelle 18, I-50014 San Domenico di Fiesole (FI); telephone: 055 4685919. E-mail: piero.gottardi@eui.eu. 
Recent economic events have made it clear that looking at financial entities in isolation, abstracting from their linkages, gives an incomplete and possibly very misleading impression of the potential impact of shocks to the financial system. In the words of Acharya et al. (2017) "current financial regulations, such as Basel I and Basel II, are designed to limit each institution's risk seen in isolation; they are not sufficiently focused on systemic risk even though systemic risk is often the rationale provided for such regulation." The aim of this paper is precisely to investigate how the capacity of the system to absorb shocks depends on the pattern of connections established among firms.

More specifically, we study the trade-off between the risk-sharing benefits to firms of higher interconnection and the costs resulting from an exposure to a larger set of sources of risk. Clearly, this trade-off must be at the center of any regulatory effort that takes a truly systemic view of the financial world. By formulating the problem in a stylized and analytically tractable framework, we can examine how the pattern of linkages among firms should optimally be tailored to the underlying shock structure. We can also shed light on the key issue of whether these normative prescriptions are consistent with the incentives of individual firms to establish linkages.

We analyze a finite environment with $N$ financial firms. In most of the paper we shall consider the case where all firms are identical ex ante, with the same level of assets and liabilities, and endowed with a risky project displaying the same probabilistic pattern of returns. Ex post, however, they will often be different since, with some probability, a shock hits one randomly selected project, decreasing the income it generates. We allow each firm to hold claims to the yield of the projects of other firms, in addition to claims on its own project. This generates financial linkages among the firms in the system. It implies that a shock hitting the project of a firm also affects all other firms linked with it, in proportion to their exposure to this firm.

When the revenue of the assets of a firm falls below its liabilities a firm defaults. The presence of linkages to other firms allows a firm to reduce the exposure to the shocks that may hit its own project, and hence to lower the probability of default in such event, thus providing risk sharing. At the same time, these linkages expose the firm to shocks hitting other firms. The precise effects of the shock depend then on the pattern of financial linkages among all the firms, that is, on the system's financial structure. 
Clearly, the maximum extent of risk sharing obtains when all firms belong to a fully connected network. This configuration, however, also exhibits the widest exposure of firms to shocks: any shock hitting a firm's project affects all firms in the system and, if the shock is large, could lead to extensive default. There are two alternative (possibly complementary) ways of reducing the possibility of contagion. One is 'segmentation,' which has firms connected only to a subset of other firms, thereby dividing the system of firms into disjoint components. 11 Naturally, this isolates the firms lying in any given component from the shocks hitting any other component. A second way is to operate on the 'dispersion of connections', that is, to modulate the exposure that any given firm has to the various firms in its component. For example, a firm could have a high level of exposure to some firms, while keeping a significantly lower exposure to the remaining ones.

One of the primary objectives of this paper is to identify the financial structure that maximizes social surplus for various properties of the shock distribution, thus solving optimally the trade-off between risk sharing and contagion. Social surplus is maximized when the expected number of defaults in the system is minimal. A very important concept in our analysis of this trade-off is the risk exposure function, which describes the probability of default of a firm as a function of the size of its exposure to another firm. We will show that the optimal pattern of firms' exposures is closely related to the curvature properties of the risk exposure function. In turn, these properties are linked to those of the probability distribution of the shocks, and in particular they depend on how the probability mass on shocks varies with their size.

We show first that, when the risk exposure function is globally convex the optimal structure exhibits full integration, with the exposure of a firm to shocks other than its own evenly divided among all other firms. Conversely, the optimum features maximal segmentation when the risk exposure is everywhere concave. To better understand this result, note that the convexity property is satisfied, for example, for shocks whose probability distribution is Pareto with relatively little mass on the tail, so that large shocks are not very likely. It is natural that in this context the optimum is primarily driven by the concern for risk sharing, with uniform exposure to all other firms. On the other hand, a Pareto distribution with a large probability mass on the tail yields a concave risk exposure function. In this case, the concern for limiting contagion prevails and the

\footnotetext{
${ }^{1} \mathrm{~A}$ component is defined to be a maximal set of firms that are all mutually connected/exposed at some positive level. See Subsection 1.3 for details.
} 
optimal structure exhibits maximal segmentation into components of the smallest size.

When instead the risk exposure function is convex for some range of exposures and concave for others (that is, neither globally concave, nor globally convex), the optimal pattern of exposures involves a compromise. To ensure some risk sharing as well as the presence of circuit-breakers that limit contagion, the optimum resorts to either intermediate degrees of segmentation or the modulation of the levels of exposure to other firms. The particular alternative chosen depends on the specific way in which changes in curvature occur (in particular on whether the risk exposure is concav $\oint^{2}$ or convex for levels of exposure close to zero). The first route introduces in fact more rigid circuit breaks in the system (yielding either full or no connection between any two firms), while the second one exhibits more flexibility in the response to shocks.

Next we examine whether the incentives of individual firms to establish linkages with other firms (so as to minimize individual default probabilities) are aligned with social optimality. To this end, we consider a simple network-formation game where each firm proposes the set of other firms to which it wants to connect. Then, for all such proposals that are jointly accepted by all those involved, the corresponding links are established and a connected component of the system is formed. We show that for those scenarios where social optimality requires polar segmentation (i.e. components of maximum or minimum size), equilibrium and socially optimal outcomes coincide. This is not so, however, when optimality prescribes intermediate segmentation. For, in this case, a conflict typically arises: in the equilibrium configuration some components are inefficiently large and one of them is inefficiently small. In the end, therefore, the default probabilities are inefficiently high.

The final part of the paper extends the analysis to the case where firms are ex-ante heterogeneous, in particular with regard to the risk characteristics of the shocks hitting the projects of the various firms. In this context, our main conclusion is that optimality requires perfect assortativity, i.e. segmentation along firms' types, with components formed by homogeneous firms with the same risk profiles of shocks.

Our model, although stylized, allows for the guidance of concrete policy advice. Because of the increase in financial firms' interconnections (see Diebold and Yilmaz 2009), it is now widely

\footnotetext{
${ }^{2}$ This property is satisfied, for example, when the shock follows a mixture of Pareto distributions with finite and infinite first moments.
} 
judged to be insufficient to rely primarily on the regulation of capital requirements ${ }^{3}$ Some authors, like Stiglitz (2010), have argued that a policy tool consisting effectively of modulating exposures within a network can be effective, and Degryse and Nguyen (2007) provide evidence about this in a concrete empirical example 4 Our paper provides some general results about how the optimal structure of the network depends on the distribution of shocks. We believe this is an important contribution since, as DTTC (2015) points out: "one of the challenges of designing direct measures is the uncertainty surrounding the level of interconnectedness that is deemed undesirable," and our model can give reasonably precise answers to that challenge.

Our approach is in marked contrast with the one currently pursued by the literature on, and the practice of, macroprudential regulation. For example, one customary way of addressing the problem is to rely on stress tests. This, essentially, amounts to simulation exercises examining the consequences for a system of interconnected institutions of being subject to a large shock (see e.g. ESMA 2016 or Kara, Tian and Yellen 2015). This does not take into account that, as we show here, a very connected network will collapse with a sufficiently large shock, while at the same time such connectedness also provides the benefit of forestalling problems with smaller, perhaps much more frequent, shocks. Thus, it is not possible to get a sense of the optimality of a particular connection structure without understanding the impact of the whole distribution of shocks 5 Another approach for assessing risks for banking systems is to simulate the impact of draws from the empirical distribution of past observed returns under the connectedness observed in the actual network (Elsinger, Lehar and Summer 2006). This is closer to what we do in this paper but, rather than looking at a specific network and distribution of shocks, we provide results that apply for general networks and shock distributions.

An additional normative aspect we have highlighted is that, when different institutions are hit by

\footnotetext{
${ }^{3}$ The Basel III agreements require higher overall equity requirements than previous rounds plus and additional capital conservation buffer of $2.5 \%$ during expansions to combat the pro-cyclicality of credit.

${ }^{4}$ In their own words, "a change from a complete structure (where all banks have symmetric links) towards a 'multiple money centre' structure (where the money centres are symmetrically linked to some banks, which are themselves not linked together) as well as a more concentrated banking market have decreased the risk and impact of contagion."

${ }^{5}$ The weight attributed to shocks of different sizes clearly depends on how social surplus is affected by the number of defaults in the system. We consider the case where social surplus only depends on the expected number of banks failing. But if the social cost of, say, ten firms defaulting were much larger than ten times the cost of one firm defaulting, the impact of very large shocks would be more important, and stress tests would be more valuable. We further discuss the effects of the consideration of alternative welfare criteria in footnotes 21 and 32 .
} 
shocks coming from different distributions, they should belong to separate components (although, as we explain in more detail later, there may not be a conflict with equilibrium behavior in this case). The intuition for this result can be grasped easily by contrasting the following two polar cases: a firm facing a distribution with little mass on the tail benefits by belonging to a very large component, whereas a firm facing a distribution with large mass on the tail benefits by being part of a minimal component. If both types of firm happen to be in the same component, at least one of them is bound to be part of a component of suboptimal size. This conclusion leads to an important policy implication, and adds a novel justification for the separation of investment and commercial banking which was enforced by the Glass-Steagall act from 1933 to 1999 $[$ In our model, this separation may prove beneficial as a way to cope with a plurality of institutions that are subject to shocks with different risk profiles. For example, it is conceivable that, for this reason, commercial banks should have a much larger component size than investment banks. In fact, several current policy initiatives aim precisely at limiting risk exposures by separating banking activities that have intrinsically different risk levels - e.g. the Volcker rule in the U.S., or the Vickers and the Liikanen proposals (see DTCC (2015)).

This normative implication does not necessarily imply the need for a regulatory intervention, as the equilibrium might already display the required separation between institutions with quite different risk profiles. But we observe that in reality those institutions are often part of the same corporation. This could of course be due to some additional factors we have ignored here, and could then be still efficient. And, yet again, it could be the unintended result of other government interventions. For example, commercial banks benefit from a lower cost of capital due to an implicit or explicit government guarantee, and investment banks want to benefit from that regulation as well. At a minimum, our result suggests that this kind of "merged risk profiles" should be studied carefully by regulators.

\footnotetext{
${ }^{6}$ The traditional view was that the separation is necessary to protect consumers from conflict of interest between these institutions, but Kroszner and Rajan (1994), for example, find little evidence for this argument.
} 


\section{Related literature}

The research on financial contagion and systemic risk $7^{7}$ is quite diverse and also fast-growing. Hence we shall provide here only a brief summary of some of the more closely related papers 8

Allen and Gale (2000) pioneered the study of the stability of interconnected financial systems. They analyze a model in the Diamond and Dybvig (1983) tradition, where a network structure with a single, completely connected component is always optimal, i.e. the one that minimizes the extent of default. Our model, in contrast, shows that a richer shock structure can generate a genuine trade-off between risk-sharing and contagion, and that both segmentation and lower dispersion of connections may be optimal. 9

Three more recent related papers are Elliott et al. (2014), Acemoglu et al. (2015), and Glasserman and Young (2015). The nature and form of the financial linkages among firms considered in these papers are partly different, as they concern not only the asset side but also the liability side of the firms' balance sheet. This, in turn, entails the presence of some amplification mechanism of the shocks hitting a firm 10 Another difference with our paper concerns the main focus of the analysis. While the aim of Elliott et al. (2014) is to characterize conditions on the structure of the network under which default cascades occur, the primary objective of ours is to characterize the optimal financial structures in diverse scenarios and their relationship with individual incentives. Acemoglu et al. (2015) shares with us the focus on the optimality of financial networks, but restricts its analysis to shock distributions that are degenerate Dirac measures concentrated on a given shock magnitude 11 Our characterization results, on the other hand, are mostly stated in terms of properties of the curvature of the risk exposure function, and hence of the cumulative distribution of

\footnotetext{
${ }^{7}$ There is also a large body of literature that studies the general problem of risk sharing in non financial contexts, largely motivated by its application to consumption sharing in poor economies that lack formal insurance mechanisms. Paradigmatic examples are the papers by Bramoullé and Kranton (2007), Bloch et al. (2008), and Ambrus et al. (2014).

${ }^{8}$ The reader is referred to Allen and Babus (2009) and to Cabrales et al. (2016) for recent surveys of the literature on financial contagion in networks.

${ }^{9}$ Babus (2016) uses a similar model but allows for the endogenous formation of links between banks, and shows that banks manage to form networks that are usually resilient to the propagation of shocks.

${ }^{10}$ See Cabrales et al. (2016) for a more general discussion of the effects of these differences in the specification of linkages. In particular, it is argued that the qualitative features of optimal financial structures are not affected.

${ }^{11}$ The papers by Glasserman and Young (2015) and Acemoglu et al. (2015) display some common features, but the former allows for continuous distributions of the shocks (as we also do). The main result of Glasserman and Young is a characterization of the parameter values and networks for which the probability of default of a firm is lower when a shock hits some its neighbors than when the firm is directly hit.
} 
shocks. These properties allow to consider a rich set of possible distributions and to show the different ways in which the optimal financial structure varies in response to the characteristics of those distributions. This richness sets us apart from all the other papers in the area 12

Another branch of the literature focuses on how financial contagion is affected by imperfect information on the shocks hitting the system. For instance, Allen et al. (2011) explore the effects on segmented and unsegmented structures of the arrival of a signal indicating that some firm in the system will have to default 13

Finally, we should also mention the large empirical and policy-oriented literature whose main objective has been to produce summary measures derived from the network of relationships among firms (mostly banks) with the aim of predicting the likelihood of systemic failures - see, for example, Battiston et al. (2012) or Denbee et al. (2011), which propose different measures of centrality in networks. Of particular interest in this respect is the paper by Elsinger, Lehar and Summer (2011) who, using Austrian data, show that correlation in banks' asset portfolios is the main source of systemic risk 14

The rest of the paper is organized as follows. Section 1 describes the economic environment and the possible patterns of linkages among firms. Section 2 characterizes the optimal financial structures for various properties of the shock distribution. Section 3 addresses the issue of network formation and the relationship between individual incentives to form linkages and social optimality. Section 4 extends the analysis to allow for heterogeneity in the distribution of the shocks that may hit different firms. Section 5 concludes. For convenience, the proofs of our results are relegated to the Appendix.

\footnotetext{
${ }^{12}$ There is also a complementary line of literature that, in contrast with the papers just mentioned, studies the issue of contagion and systemic risk in the context of large networks. While most papers follow a numerical approach, based on large scale simulations (see Leitner (2005) and Gai et al. (2011)), Blume et al. (2011) use the mathematical theory of random networks to study the optimal average degree of the network and its consistency with individual incentives to create or destroy links.

${ }^{13}$ On a similar line, Caballero and Simsek (2013) and Alvarez and Barlevy (2014) study how informational contagion can amplify the contagion resulting from mutual exposure between firms.

${ }^{14} \mathrm{~A}$ related emphasis on cross-ownership and investment has been pursued as well by the literature on "balance sheet effects" to understand the Asian financial turmoil in the late 90's (Krugman 1999) as well as the current crisis (Ahrend and Goujard 2011). These latter approaches are very much in line with our model, which precisely highlights portfolio correlation as the key driver of default risk.
} 


\section{The Model}

\subsection{The Environment}

Our benchmark scenario considers an environment with $N$ ex ante identical financial firms (say, banks). We assume $N$ is even. Each firm $i$ has one project which yields a gross return given by a random variable $\widetilde{R}_{i}$ as follows. With probability $1-q$ the return equals its 'normal' value $R$, while with probability $q$ the firm's investment is hit by a shock and its return equals $R-\widetilde{L}_{i}$, where $\widetilde{L}_{i}$ is a positive valued random variable with the same distribution for all $i$. On the liability side, every firm faces claims by outside investors (e.g. deposit or bond holders) equal to $M$, a parameter of the model 15

We model financial linkages by allowing each firm to hold claims to the returns obtained from the projects of other firms, together with a fraction of the claims on its own project. More precisely, for any $i, j \in \mathcal{N}=\{1, \ldots, N\}$, let us denote by $a_{i j} \geq 0$ the fraction held by firm $i$ of the outstanding amount of claims to the yield of the project of firm $j$. Hence the pattern of asset holdings across the $N$ firms in the economy can be described by a matrix $A$ of the following form:

$$
A=\left(\begin{array}{cccc}
a_{11} & a_{12} & \cdots & a_{1 N} \\
a_{21} & a_{22} & \cdots & a_{2 N} \\
\vdots & \vdots & \ddots & \vdots \\
a_{N 1} & a_{N 2} & \cdots & a_{N N}
\end{array}\right)
$$

The return on the assets owned by any given firm $i$ is then a weighted average of the yield of the firm's own project and the yields of the projects of the other firms. Subtracting from this the value of the firm's liabilities, given by $M$, we obtain the following expression for the net financial position of firm $i$ :

$$
\sum_{j=1}^{N} a_{i j} \widetilde{R}_{j}-M
$$

Hence inter-firm linkages arise through the asset side of the firms' balance sheet, since the value

\footnotetext{
${ }^{15}$ We can think of these liabilities as issued to raise external funds to finance the firm's project. See Cabrales et al. (2014) for the analysis of the case where $M$ is endogenously determined and depends on investors' preferences and firms' default probabilities.
} 
of the assets of firm $i$ may be related in general to the value of the assets of other firms $j \neq i$. In contrast, note that no linkage is present on the liability side.

When the realization of the return on the assets held by firm $i$ falls short of $M$, that is the expression in (2) has a negative sign, the firm defaults. Default is assumed costly: in addition to liquidation costs, there are opportunity costs deriving from the fact that a defaulting firm stops operating, and hence it loses any future earnings possibility.

\subsection{Shock structure}

We specify now some properties of the probability distribution of the shock $\widetilde{L}_{i}$ that may hit the yield of the project of firm $i$. Conditionally on such shock hitting firm $i$, with probability $\pi$ the shock is 'small' (labeled $s$ ) and the project experiences a loss of size $\underline{L}$. With probability $1-\pi$ the shock is 'big' (labeled $b$ ), and is described by a random variable $\widetilde{l}$, with support $\underline{16}[\underline{L}, \infty)$ and cumulative distribution function $\Phi(l)$. Summarizing, the gross return on the project of an arbitrary firm $i$ is:

$$
\widetilde{R_{i}}=\left\{\begin{array}{l}
R \text { with prob. } 1-q \\
R-\underline{L} \text { with prob. } q \pi \\
R-\widetilde{l} \text { with prob. } q(1-\pi)
\end{array}\right.
$$

For the risk of default to be an issue, we assume:

A1. (i) $R(1-q)>M$,

(ii) $R-\underline{L}<M$.

The first inequality ensures that a firm's project is viable, that is, its expected return exceeds what must be paid to lenders. On the other hand, the second inequality implies that if a firm can only draw on the revenue generated by its project, it is surely unable to pay depositors (and hence must default) whenever a shock, whether small or large (since $\tilde{l} \geq \underline{L}$ ), hits its return.

\footnotetext{
${ }^{16}$ The assumption that the support of $\widetilde{l}$ is unbounded implies that there is positive probability of a sufficiently large shock that would lead to all the firms defaulting, if they were all connected to each other. The analysis could have been equivalently carried out if we had truncated the distribution at any upper bound $\bar{L}>2(N-1)(R-M)$ (with $\bar{L} \leq R$ in order to be consistent with limited liability) and all the mass beyond $\bar{L}$ were shifted to this value. All our results would still hold, with only a qualification that we explain in Footnote 27 We adopted the current specification purely for simplicity of the analysis.
} 
We assume that shocks are rare and thus at most one project is hit by a shock. This can be motivated by postulating that, even if shocks hit projects in a stochastically independent manner, the probability $q$ that a shock hits any given one is so low that the probability that two or more shocks arrive in a single period is of an order of magnitude that can be ignored 17

On the nature of those shocks, we make the following key assumption:

A2. (i) $\pi>N(1-\pi)$,

(ii) $\frac{1}{2}(R-\underline{L})+\frac{1}{2} R>M$.

Part (i) of the above assumption says that $s$ (small) shocks are significantly more likely than $b$ (big) ones. In particular, it is more likely that a given firm is hit directly by an $s$ shock than a $b$ shock hits any firm in the system. On the other hand, part (ii) ensures, for reasons that will become clear in the next section, that the presence of sufficiently strong linkages to other firms allows a firm to fully insure against the $s$ shocks, i.e. guarantees that the firm never defaults when an $s$ shock hits any of the firms.

\subsection{Interfirm Linkages and Financial Structures}

The matrix $A$ describes the pattern of financial linkages among the $N$ firms. As we see from (2), when $a_{i j}>0$, firm $i$ is exposed to the shocks hitting the return on firm $j$ 's project. The elements of $A$ specify not only which firms have a financial linkage to any given firm $i$, but also the strength of all these linkages. Formally, we can think of $A$ as the adjacency matrix of a weighted directed network, with $a_{i j}$ representing the intensity of the link, or the exposure, of $i$ to firm $j$. To fix ideas it may help to conceive the reciprocal exposure between two firms as the result of exchanges of assets between the firms 18

\footnotetext{
${ }^{17}$ As an extreme formalization of this idea, we could model time continuously and assume that the arrivals of small and big shocks to each firm are governed by independent Poisson processes with fixed rates $\pi$ and $1-\pi$, respectively. Then, the probability that two shocks arrive simultaneously is zero.

${ }^{18}$ We can also interpret the linkages with higher intensity as describing direct linkages and those with lower intensity as describing indirect linkages. See Cabrales et al. (2014) for a formal derivation of possible patterns of linkages arising from the iteration of exchanges of assets among firms located in a network. In such setup, if we let $B$ be a matrix describing the pattern of exchanges of assets among firms that are directly connected, we obtain $A=B^{T}$ for some $T>1$ that describes the number of iterations of asset exchanges conducted by neighboring firms. In a stylized manner, the matrix $A$ can so be viewed as the outcome of a process of securitization implemented through multiple rounds of asset trade.
} 
The elements of $A$ can take any value between 0 and 1 . To ensure a balance among the positions of different firms, it is natural to require that not only the columns but also the rows 19 of $A$ sum to 1 :

$$
\sum_{j=1}^{N} a_{i j}=\sum_{i=1}^{N} a_{i j}=1 \quad i, j=1,2 \ldots, N
$$

The presence of financial linkages allows a firm to reduce its exposure to the shocks that may hit the return on its own project and hence lower the probability that a firm defaults when its project is hit by a shock. At the same time, these linkages imply that the firm is also exposed to the shocks hitting firms with which the firm is thus connected. This may generate a sort of contagion in the following sense: a shock hitting the project of any firm can affect many other firms by decreasing the value of the assets they hold and hence, possibly, induce widespread default.

More precisely, when a shock of size $L$ hits the return on the project of firm $i$, this firm defaults if

$$
a_{i i}(R-L)+\sum_{j \neq i}^{N} a_{i j} R<M
$$

When such a shock hits the return on the project of some other firm $k \neq i$, firm $i$ defaults if

$$
a_{i k}(R-L)+\left(a_{i i}+\sum_{j \neq i, k}^{N} a_{i j}\right) R<M
$$

Hence the lower $a_{i i}$, the less likely expression (5) is satisfied. At the same time, given (4), this also means that it is more likely that (6) holds. Therefore, in the environment considered, there is a trade-off between risk-sharing and contagion.

REMARK 1 In our model the linkages among firms capture the fact that they are exposed to common shocks through the shared ownership of claims on the yields of the different projects. An important consequence of this feature is that the default of no firm has, per se, an effect on the solvency of other firms. Therefore, the possibility of contagion from a large shock hitting one firm comes only from the correlation in returns induced by the assets commonly held in firms' portfolios 20

\footnotetext{
${ }^{19} \mathrm{~A}$ condition of this kind would be needed if we think of the firms' cross-holdings as resulting from exchanges of assets among them and, due to symmetry, the projects of all firms display the same ex ante pattern of returns.

${ }^{20}$ Lazear (2011), in a widely discussed Wall Street Journal article, refers to this mechanism as the 'popcorn' effect. He contrasts it to the 'domino' effect, where it is the default of one firm that triggers the contagion to other firms.
} 
In the environment described, the financial structure, as described by the matrix $A$ determines how shocks hitting any firm are distributed over the whole set of firms. As long as no firm defaults, this only entails a redistribution of resources across firms and between the owners of the different firms. When defaults occur there is again a redistribution of resources, now between creditors and owners of the firms as well. But since defaults are costly, the amount of available resources is also negatively affected, the more so the larger the number of firms defaulting. When all investors are risk neutral we can assess the performance of a financial structure in terms of social surplus, given by the sum of the expected revenue generated by the firms' assets, less the costs of default. Since these costs are the same for all firms, we can then say that welfare, as measured by total surplus, is maximal when the expected number of firms defaulting is minimal 21 Also, any firm maximizes its value, in the presence of risk neutral investors, when it minimizes its probability of default.

One of the primary objectives of this paper is to characterize the optimal financial structures in the face of various properties of the probability distribution $\Phi($.$) of the big shocks that may$ hit the system. The set of possible financial structures is quite large. In order to emphasize the key dimensions in which they can differ, it is useful to introduce the following three notions: (1) externalization, (2) segmentation, and (3) dispersion of linkages.

Externalization refers to the exposure to the project of other firms, relative to the exposure to the firm's own project. For an arbitrary firm $i$, this is measured by $1-a_{i i}$. Assumptions A1(ii) and A2(i) imply that a firm with no linkages always defaults when hit by an $s$ shock, and that $s$ shocks are much more likely than $b$ shocks. It then follows from A2(ii) that any firm $i$ can completely avoid default when $s$ shocks occur by establishing some linkages to other firms, provided $1-a_{i i}$ is sufficiently close to $1 / 22^{22}$ Given our aim of characterizing optimal financial structures, in what follows we will focus our attention on the set of structures that satisfy this condition, and display

\footnotetext{
${ }^{21}$ In our specification welfare is only affected by the expected number of defaults in the system. One could argue however that the variability of the number of defaults also matters, as the occurrence of multiple contemporaneous defaults is problematic (e.g. because of unmodeled resales externalities). See, e.g. Beale et al. (2011) for the proposal of a criterion to evaluate financial systems based on the minimization of a "systemic cost function" defined as the expectation of a convex function of the number of defaults in the system. This would have the effect of making contagion, and hence the formation of linkages among firms, costlier but we expect most of our qualitative findings on optimal financial structures to extend to such a specification, with one exception, to which we refer in footnote 32.

${ }^{22}$ More specifically, this statement always holds if, for any given $i, a_{i j}(R-\underline{L})+\left(1-a_{i j}\right) R \geq M$ for all $j$. When $a_{i i}=1 / 2$, it follows from (4) that $a_{i j} \leq 0.5$ for all $j$ and hence $a_{i j}(R-\underline{L})+\left(1-a_{i j}\right) R \geq 0.5(R-\underline{L})+0.5 R$. Assumption A2(ii) then implies that the term on the right hand side of the last inequality is strictly greater than $M$.
} 
a common externalization of risk 23

$$
1-a_{i i}=1-\alpha \text { for all } i \text {, for some given } \alpha \text { close to } \frac{1}{2} \text {. }
$$

Even though all the financial structures we consider have the same diagonal terms, they can nevertheless differ widely in their off-diagonal terms. Hence they may differ widely in the ability firms have to withstand the indirect effects of $b$ shocks, that is, when such shocks hit the project of some other firm in the system. Thus, while the term on the left hand side of (5) - which specifies the consequences on the value of the firm's assets of being hit directly by a shock - depends only on the main-diagonal entries and has the same value under all the network structures considered, the term on the left hand side of (6) - which pertains to indirect effects - depends on the off-diagonal terms $\left(a_{i k}\right)_{i \neq j}$ and its value will vary across alternative network structures.

Segmentation describes how the system is divided into independent components. In the network literature a component is identified with a maximal set of nodes that are mutually connected, directly or indirectly. In our context, for $i$ to be connected to $j$ there has to be a sequence of firms starting at $i$ and ending at $j$ with the property that any firm $k$ in the sequence has positive exposure to its immediate successor $l$ in the sequence, so that $a_{k l}>0$. A set $\mathcal{D} \subset \mathcal{N}$ is then connected if, for every ordered pair $i, j \in \mathcal{D}, i$ is connected to $j$; more formally:

$$
\forall i, j \in \mathcal{D}, \exists\left\{k_{1}, k_{2}, \ldots, k_{r}\right\} \text { s.t. } a_{k_{q}, k_{q+1}}>0 \text { for all } k_{q}(q=1,2, \ldots, r-1) \text {, with } k_{1}=i \text { and } k_{r}=j \text {. }
$$

A component is a subset $\mathcal{C} \subset \mathcal{N}$ that is maximally connected, i.e. it is itself connected and there exists no $\mathcal{C}^{\prime} \supsetneqq \mathcal{C}$ that is also connected.

In what follows we shall also require that every component in the system is such that every two firms lying in it are directly connected with an intensity that is bounded below by some given $\varepsilon$, which can be arbitrarily small (albeit positive). That is, they are in a situation of mutual exposure:

$$
\exists \varepsilon>0 \text { s.t. } \forall i, j \in \mathcal{C}, a_{i j} \geq \varepsilon
$$

The motivation for this requirement derives from our interpretation of the nature of the relationship

\footnotetext{
${ }^{23} \mathrm{~A}$ further optimization with respect to the level of $\alpha$ (subject to the constraint in footnote 22 would not yield extra insights with respect to the issue we are mainly concerned with here, namely, the effect of contagion on default.
} 
between firms $i$ and $j$, when firm $i$ is exposed to the return of the project of firm $j$. In that case $a_{i j}>0$, and this means that firm $i$ gains access to information and the possibility to monitor the activities of $j$. This naturally would generate an interest in $j$ to understand the motivations and the running of firm $i$. To achieve this, firm $j$ could acquire some claims to the project of $i$ so that $a_{j i} \geq \varepsilon$. These informational and incentive issues arise in the real world, and are indeed often dealt with through the joint participation in ventures of shared interest. This is precisely the point made by Berglöf and Perotti (1994), who assert that, in business networks, firms resort to such schemes because "[t]he reciprocal allocation of control rights supports cooperation and mutual monitoring among managers." Similar considerations apply when firm $i$ is exposed to $j$, and $j$ is exposed to $k$. Then firm $i$ would also like to monitor and gain information about $k$, which can be attained if $a_{i k} \geq \varepsilon$.

Condition (8) ensures that, given the pattern of exposures of an individual firm $i$, as described by the corresponding row of the matrix $A$, the component where that firm lies is of the smallest possible size ${ }^{24}$ This condition, together with the balance condition (44), implies there is an interdependence between the pattern of exposures of the different firms. This creates a need for coordination both within and across components since the exposures of any individual firm cannot be considered in isolation from those of other firms.

Let us denote by $K_{i}$ the number of firms to which any given firm $i$ is directly connected. Condition (8) implies that $K_{i}$ takes the same value for each firm $i$ lying in a given component. The size of the component is then given by $K_{i}+1$. Therefore the pattern of segmentation in the system is determined by the number $C$ of components and the size of each of them. In the extreme cases of no segmentation we have $K=N-1$. In the case of maximal segmentation we have $K_{i}=1$ for all $i$, because by condition (7) every firm must have a connection to at least another firm, and thus the minimal component size is 2 . Rows and columns of $A$ can always be rearranged so that this matrix has a block diagonal structure, with the blocks corresponding to the network components.

The dispersion of exposure within any component (say, of size $K+1$ ) describes the pattern

\footnotetext{
${ }^{24} \mathrm{~A}$ very simple example may be used to illustrate the point. Suppose that the pattern of exposures of a firm is characterized by positive exposure to only one other firm. This can be achieved by having a component with two firms mutually exposed to each other and not connected to the rest. Alternatively we could have each firm $i \in \mathcal{N}$ exposed to, say, firm $i+1$, with the highest-index firm $N$ exposed to firm 1 . This would be a component of size $N$ but would contradict condition (8).
} 
of connection that each individual firm establishes with the rest of firms lying in its component. Formally this is captured by the entries off the main diagonal of the submatrix $A_{K}$, that is the $K+1$-dimensional adjacency matrix that includes only the entries corresponding to the $K+1$ firms in the component. In the case of uniform exposure (that is, maximal dispersion) the off-diagonal entries are all equal to $(1-\alpha) / K$. Alternative configurations display lower levels of dispersion, and exhibit some inter-firm asymmetries with the exposure to some of the firms in the component being higher than the exposure to others.

With no segmentation and maximal dispersion we have $a_{i j}=(1-\alpha) /(N-1)$ for all $i, j$, so that every firm is equally exposed to every other firm in the system. In this situation we have maximal risk sharing, but also maximal possibility of contagion. A first way in which the spread of contagion can be limited is via segmentation, so that $a_{i j}=0$ for some $j$. But this also means that, whenever $a_{i j}>0$ its average value is generally greater than $(1-\alpha) /(N-1)$, the more so the greater is segmentation. Hence the exposure of a firm is concentrated on a subset of firms, which in turn entails a lower extent of risk sharing. A lower dispersion of the connections provides another way in which contagion can be limited: in that case the exposure of a firm is also unevenly distributed, but this happens by modulating the intensity of linkages within a component rather than by dividing the system into disjoint components. Note also that our specification allows for asymmetries in the patterns of exposure of different firms, as different components may be of different sizes and exhibit different dispersions of connections.

\section{Optimal Financial Structures}

In this section we will determine the optimal financial structure, and hence the degree of segmentation and the intensity of linkages within each component that allows to minimize the expected number of defaults in the system, for different properties of the cumulative distribution function $\Phi($.$) of the b$ shocks that may hit firms. To find the optimal financial structure we proceed as follows.

From (6) we see that, when a shock hits some firm $k$, firm $i \neq k$ defaults if the (random) 
magnitude $L$ of this shock is sufficiently large relative to the size of the exposure of $i$ to $k$, that is

$$
L>\frac{R-M}{a_{i k}}
$$

Hence the probability that $i$ defaults, conditionally on the event that firm $k$ is hit by a $b$ shock, is:

$$
1-\Phi\left((R-M) / a_{i k}\right)=: F\left(a_{i k}\right) .
$$

In the analysis that follows we will conveniently state the optimization problem yielding optimal financial structures in terms of the function $F($.$) , which describes the risk of default for firm$ $i$ induced by its exposure to the shocks hitting other firms. For short, we will refer to $F($.$) as$ the risk-exposure function. Note that the function $F(\cdot)$ is always weakly increasing and satisfies $F(0)=0$.

We proceed in two steps. First, we find the optimal pattern of connections in a component of size $K+1$, for any $K=1, . ., N-1$, that is the one minimizing expected defaults in a component. Using the fact that a $b$ shock can hit any of the firms with equal probability, this is obtained as a solution of the following problem:

$$
\begin{aligned}
{[\mathbf{P}(K)] \quad V(K)=\min _{\left[\left(a_{i j}\right)_{j \neq i}\right]_{i=1}^{K+1}} \frac{1}{K+1} \sum_{i=1}^{K+1} \sum_{j \neq i} F\left(a_{i j}\right) } & \text { s. t. } \\
\sum_{j \neq i}^{K+1} a_{i j} & =\sum_{i \neq j}^{K+1} a_{i j}=1-\alpha, \quad \forall i \neq j(10) \\
a_{i j} & \geq \varepsilon^{\prime} \quad \forall i \neq j
\end{aligned}
$$

The expression appearing in the objective function specifies the expected fraction of firms defaulting in a component of size $K+1$ when indirectly hit by a $b$ shock, conditionally on such a shock hitting a firm in the system. To minimize this expression amounts to minimizing the expected number of defaults in the component since, as we noticed, the probability that a firm defaults when directly hit by a $b$ shock is the same across all admissible financial structures. Constraint 10$)$ of problem $[\mathbf{P}(K)]$ restates the balance condition (4) while condition (11) requires that, within the component, every pair of firms are mutually and positively exposed to one another as per condition (8). We denote 
then by $V(K)$ the value of the objective function at a solution of problem $[\mathbf{P}(K)]$, constituting the 'optimal value of a component of size $K+1$ '.

In the second step we find the optimal segmentation, that is, the optimal partition of the $N$ firms into connected components:

$$
\begin{array}{r}
\min _{K_{c} \in\{1, . ., N-1\}, c=1, . ., C ; C \in\{1, . ., N / 2\}} \sum_{c=1}^{C} V\left(K_{c}\right)\left(K_{c}+1\right) \\
\sum_{c=1}^{C}\left(K_{c}+1\right)=N
\end{array}
$$

where the expression appearing in the objective function gives the expected number of firms in the system that default when indirectly hit by a $b$ shock (again, conditionally on such a shock hitting a firm in the system).

To derive the solution of problem $[\mathbf{S P}]$, it will often be convenient to consider the following individual optimization problem:

$$
\begin{aligned}
& \text { [IP] } \min _{\left(a_{i j}\right)_{j \neq i}} \sum_{j \neq i} F\left(a_{i j}\right) \quad \text { s. t. } \\
& \sum_{j \neq i}^{N} a_{i j}=1-\alpha \\
& a_{i j} \geq 0, \quad \forall j \neq i
\end{aligned}
$$

This problem consists in the choice of the pattern of exposures of firm $i$ to other firms in the system - that is, of the off diagonal elements of the $i$-th row of matrix $A$ - that minimizes the probability of default of firm $i$ when indirectly hit by a $b$ shock. As argued in Section 1.3 , the value of firm $i$ is maximal when the firm's probability of default is minimal. Problem $[\mathbf{I P}]$ will then also play a role in the next section when we investigate the incentives of individual firms to establish linkages at an equilibrium of a network-formation game.

Note that both the objectives of the individual $[\mathbf{I P}]$ and the social optimum problem $[\mathbf{S P}]$ are the minimization of expected defaults, and minimizing the probability of default of any single firm ensures that the expected number of defaults in the system is also minimized 25 The key difference

\footnotetext{
${ }^{25}$ Formally, if $a_{i j}$ for $i \neq j$ is such that $\sum_{j \neq i} F\left(a_{i j}\right)$ is minimal for all $i$ clearly ensures that $\sum_{i=1}^{N} \sum_{j \neq i} F\left(a_{i j}\right)$ is also minimal.
} 
between $[\mathbf{S P}]$ and $[\mathbf{I P}]$ lies in the fact that in the second optimization problem the pattern of exposures of each individual firm is chosen so as to minimize the default probability of this firm, abstracting from the constraints that relate such values to those of other firms. These are the constraints that appear in the second condition of $(10)$ and in 111 of subproblem $[\mathbf{P}(K)]$, that is, the balance condition (capturing the fact that linkages are formed via trades among firms and, in order to carry out any desired asset exchange, the two parties must agree to it) and the condition that connected firms must be in a situation of mutual exposure. These constraints are ignored here, hence the values obtained by solving $[\mathbf{I P}]$ for each firm $i$ may not be feasible. In addition, problem $[\mathbf{S P}]$ requires that the $N$ firms in the system are allocated to components so as to minimize expected defaults in the system and this objective may be in conflict with that of minimizing defaults of individual components.

Thus if (i) the values of $a_{i j}$ solving [IP] for each $i$ satisfy the missing constraints of subproblem $[\mathbf{P}(K)]$ and (ii) the firms in the system can be arranged into components so as to implement the solution of $[\mathbf{I P}]$ for every $i$, then the solution of problem $[\mathbf{S P}]$ is obtained from the solution of the simpler problem $[\mathbf{I P}]$. As we will see in what follows, (i) and (ii) are satisfied in several cases, but not always. When (i) and/or (ii) do not hold, a solution of $[\mathbf{S P}]$ cannot be obtained in this simpler way.

Having stated formally the social optimization problem, we proceed to characterize optimal financial structures for a variety of shock scenarios. Firms' exposures are determined so as to optimally address the trade-off between achieving a high level of risk sharing and reducing the risk of contagion. We will show that, for classes of shock distributions satisfying appropriate regularity properties the optimum exhibits very clear-cut and polarized features, where a firm is either minimally exposed to others or has a uniform exposure to all other firms. However, for shock distributions with richer patterns the optimum features intermediate degrees of segmentation and/or some varying modulation of the intensity of connections. The conditions will be stated in terms of properties of the curvature of the risk-exposure function $F(\cdot)$ and, as discussed below in Remark 2, are closely related to the properties of the curvature of the distribution $\Phi($.$) .$

In order to understand the determinants of these different optimal outcomes, it is useful to start by singling out some specific conditions on the shock distribution that are required for different 
patterns of exposures to arise at an optimum. This is the objective of the following:

Proposition 1 Suppose $F(\cdot)$ is twice continuously differentiable and let $\left(a_{i j}^{*}\right)_{j \neq i}$ be the individually optimal pattern of exposures (i.e., $\left(a_{i j}^{*}\right)_{j \neq i}$ is a solution of $\left.[\boldsymbol{I P}]\right)$.

[1] If $\left(a_{i j}^{*}\right)_{j \neq i}$ features a zero level of exposure to some firms (that is, $a_{i j}^{*}=0$ for some $j \in \mathcal{N}$ ), then $F^{\prime}(0) \geq F^{\prime}\left(a_{i k}^{*}\right)$ for all $a_{i k}^{*}>0$.

[2] If $\left(a_{i j}^{*}\right)_{j \neq i}$ has $p \geq 2$ distinct, positive levels of exposure to other firms (that is, there are $p$ elements $a_{i j_{l}}^{*}, j_{l} \in \mathcal{N}, l=1, . ., p$ such that $a_{i j_{l}}^{*}>0$ for all $l$ and $a_{i j_{l}}^{*} \neq a_{i j_{m}}^{*}$ for all $l, m \in\{1, \ldots, p\})$, then the concavity of $F($.$) must change at least p-1$ times.

[3] Suppose $\left(a_{i j}^{*}\right)_{j \neq i}$ features the same level of exposure $\bar{a}_{t}$ towards $n_{t}>0$ other firm $2^{26}$, and $k \geq 2$ distinct levels of exposure overall. If $\bar{a}_{v}=0$ for some $v \in\{1, \ldots, k\}$ and $\bar{a}_{t}<1-\alpha$, we must have $F^{\prime}\left(\bar{a}_{t}\right)=F\left(\bar{a}_{t}\right) / \bar{a}_{t}$ for all $t \neq v$. If instead $\bar{a}_{t}>0$ for all $t=1, . ., k$, then $F^{\prime}\left(\bar{a}_{t}\right)=F^{\prime}\left(\bar{a}_{v}\right)=\left(F\left(\bar{a}_{t}\right)-F\left(\bar{a}_{v}\right)\right) /\left(\bar{a}_{t}-\bar{a}_{v}\right)$ for all $t, v \in\{1, \ldots, k\}$.

The above result highlights some necessary conditions that must be satisfied to obtain certain properties of the individually optimal pattern of exposures. First, [1] asserts that if it is optimal to have zero exposure to some firm, then the marginal effect on the probability of default of increasing exposure to this firm must be higher than that of increasing exposure to any other firm (for which there is positive exposure). Next [2] indicates that multiple distinct levels of positive exposure can only arise if the risk-exposure function $F(\cdot)$ does not have a uniform curvature. For, only if there is a change in such curvature, the necessary and sufficient conditions for optimality can be satisfied at different exposure levels. Finally, [3] addresses the case where the optimum features the same level of exposure towards different firms. For this what is needed is that the effect on risk of adjustments in the intensive margin (as captured by the derivative of the function $F(\cdot)$ ) is the same as that of changes along the extensive margin (expressed by the average change of risk per unit of exposure $\left(F\left(\bar{a}_{t}\right)-F\left(\bar{a}_{v}\right)\right) /\left(\bar{a}_{t}-\bar{a}_{v}\right)$ between two distinct levels $\left.\bar{a}_{t}, \bar{a}_{v}\right)$.

In Proposition 1, as well as in all other results that follow, the conditions are stated in terms of properties of the risk-exposure function $F(\cdot)$. This function operates as a useful reduced-form

\footnotetext{
${ }^{26}$ Here, and throughout the paper, we shall ignore for simplicity the constraint that $n_{t}$ and, more generally the size $K+1$ of a component, should be an integer. In the mathematical derivations we allow then $n_{t}$ to be real positive numbers.
} 
summary of all the properties of the cumulative distribution function $\Phi($.$) of the b$ shocks (or its density $\phi($.$) , when it exists) that matter for optimal financial structures. The definition of F(\cdot)$ in equation (9) clearly shows how it is related to the function $\Phi($.$) , and so are its curvature properties$ that, in the end, shape the optimal exposure pattern of a firm. For the sake of completeness, such a relationship is spelled out in the following remark, under the convenient assumption of differentiability (see the Appendix for the details).

REMARK 2 The risk exposure function $F\left(a_{i j}\right)$ is convex for all $a_{i j}>0$ if, and only if, for all $x>0$, we have:

$$
\Phi^{\prime \prime}(x) \leq-2 \frac{\Phi^{\prime}(x)}{x}
$$

That is, the cumulative distribution function $\Phi(\cdot)$ is sufficiently concave. Also, the convexity of $F(\cdot)$ implies the log-concavity of $\Phi(\cdot)$ and, conversely, the log-convexity of $\Phi($.$) implies the concavity of$ $F(\cdot)$.

We now proceed to establish some sufficient conditions for alternative exposure configurations to be optimal, as a solution of the individual problem [IP] and/or of the social problem [SP]. The analysis is organized in five different results, Propositions 2, 6. As anticipated, we will determine to what extent, depending on the properties of the probability distribution of the shocks, it is optimal to reduce risk-sharing so as to limit contagion. We will also find whether this is best achieved by increasing segmentation, or rather by lowering dispersion.

We begin in Proposition 2 by considering the case where the function $F\left(a_{i j}\right)$ is either uniformly convex or uniformly concave. As explained in Remark 3 below, the contrast between such (uniform) convexity/concavity represents a straightforward generalization of a dichotomy that often arises in the context of Pareto (or power-law) distributions between those with finite or infinite first-order moments.

Proposition 2 If the risk exposure function $F(\cdot)$ is strictly convex, the individually and socially optimal pattern of exposures $\left(a_{i j}^{*}\right)_{j \neq i}$ of any firm $i$ is given by

$$
a_{i j}^{*}=\frac{1-\alpha}{N-1} \quad \text { for all } j \neq i
$$


so there is a unique component with each firm having uniform exposure to all other firms. If instead $F(\cdot)$ is strictly concave, $\left(a_{i j}^{*}\right)_{j \neq i}$ is characterized by

$$
a_{i j}^{*}=1-\alpha \quad \text { for some for some } j \neq i, \text { and } a_{i k}^{*}=0 \text { for all } k \neq i
$$

and at the social optimum there are $N / 2$ components of minimal size 2 .

The proposition establishes that, when $F(\cdot)$ displays a uniform curvature, the optimal pattern of exposures for any firm is given by one of the two following extreme configurations: all the exposure concentrated on a single other firm, or evenly divided among all other firms. Hence in this case, whether the consideration of the benefits of risk sharing or that of the risks of contagion prevails at an optimum simply depends on the curvature of the risk-exposure function. The intuition for this result is straightforward. If the function $F(\cdot)$ is convex, the minimization of the probability of default requires that each firm be equally exposed to all other firms, since otherwise any rearrangement bringing the levels of exposure closer to each other would be preferred. When instead $F(\cdot)$ is concave, the opposite considerations apply: spreading the exposure levels, thus increasing the asymmetry in the pattern of exposure to other firms, always yields a lower value of the default probability. Hence at the optimum the external exposure of each firm is fully concentrated on a single other firm.

It is instructive to compare the sufficient conditions stated in Proposition 2 with the necessary ones highlighted by Proposition 1. First, note that both uniformly convex and uniformly concave functions do not satisfy the condition stated in part [2] of Proposition 1, and hence multiple distinct levels of positive exposure can be ruled out for either case. The condition in part [3] of Proposition 1 is also inconsistent with both kinds of uniform curvature, which implies that the same level of positive exposure towards a subset of other firms cannot be optimal, thus (external) exposure must be either concentrated on a single firm, or equally divided among all other firms. Finally, the condition appearing in part [1] of Proposition 1 is incompatible with the function $F(\cdot)$ being uniformly concave, hence in this case we cannot have a zero level of exposure to other firms. These observations, albeit simple, fulfill a twin role. First, they help us to put in a more general perspective the conditions underlying the dichotomous conclusion of Proposition 2. Moreover, perhaps more importantly, they tell us how departures from the uniform curvature of the risk-exposure function 
may lead to intermediate levels of segmentation and/or of dispersion of exposures being the optimal way to achieve risk sharing while limiting contagion.

Proposition 2 also claims that, in the environment considered, the socially optimal pattern of exposures implements for each firm the one obtained as a solution of problem [IP] for all $i$ and hence the socially and individually optimal patterns of financial linkages coincide. We show in fact that we can always arrange the firms in the system into components of minimal size 2 or maximal size $N$, as the solution of $[\mathbf{I P}]$ prescribes, and so as to satisfy the additional feasibility constraints of problem $[\mathbf{S P}]$.

To illustrate the relationship between the properties of the curvature of the risk-exposure function $F($.$) and the distribution of the probability mass over large and small realizations of the shocks,$ it is useful to consider the particular case in which the shocks are Pareto distributed.

REMARK 3 Suppose the $b$ shock $\widetilde{l}$ is Pareto distributed on $[\underline{L}, \infty)$ with continuous density

$$
\phi(l)=\gamma \frac{\underline{L}^{\gamma}}{l \gamma+1}
$$

Straightforward computations then show that, for any $a_{i j} \in(0,1]$, we have:

$$
\begin{aligned}
F\left(a_{i j}\right) & =1-\int_{\underline{L}}^{(R-M) / a_{i j}} \phi(l) \mathrm{d} l \\
& =1+\underline{L}^{\gamma}\left(\left(\frac{a_{i j}}{R-M}\right)^{\gamma}-\frac{1}{\underline{L}^{\gamma}}\right)=a_{i j}^{\gamma}\left(\frac{\underline{L}}{R-M}\right)^{\gamma}
\end{aligned}
$$

Therefore if $\gamma<1$, and hence the Pareto distribution has a sufficiently high mass on large realizations that its first-order moments are unbounded, the function $F(\cdot)$ is uniformly concave. Alternatively if $\gamma>1$, and thus the Pareto distribution has less mass on the tail and the first-order moments are bounded, the function $F(\cdot)$ is convex.

In view of the previous remark, Proposition 2 readily leads to the following corollary for the case where $b$-shocks are Pareto-distributed.

Corollary 1 Suppose the b-shocks are Pareto distributed with density given by (16). Then, the individually optimal pattern of exposures is given by (15) if $\gamma<1$, and by (14) if $\gamma>1$. 
The preceding corollary allows us to interpret the clear-cut conclusion of Proposition 2 in a particularly transparent manner if we restrict attention to a context with Pareto-distributed $b$ shocks. In this case, the key consideration is whether the decay parameter $\gamma$ is higher or lower than 1 , which simply translates into whether the expected shock is finite or infinite. In the first case, which can be heuristically described as one with little mass on the tail, the risk exposure to other firms can be regarded as comparatively safe since the probability mass associated to smaller shocks is relatively high. This leads to the conclusion that at the optimum there should be no segmentation, with a single risk-sharing component including all firms and uniform exposure to all of them. Instead, if the decay parameter is below 1 (large mass on the tail), the larger shocks are relatively more likely and the opposite desire to protect against external risk becomes preeminent. Therefore, segmentation should be maximal, to limit exposure to other firms and so contagion.

We show next that when the risk exposure function exhibits changes in its curvature, the optimal tradeoff between risk-sharing and contagion may not always result in extreme segmentation and dispersion outcomes.

First, the following result shows that an intermediate level of segmentation, where a firm is exposed to a subgroup of other firms of intermediate size (more than one but less than $N-1$ ), is individually optimal when firms face simple mixtures of Pareto distributions with finite and with infinite expected values.

Proposition 3 Suppose the distribution of $\widetilde{l}$ is given by a mixture of Pareto distributions on $[\underline{L}, \infty)$, with continuous density $\phi(l)=p \gamma_{1} \frac{L^{\gamma_{1}}}{l^{\gamma_{1}+1}}+(1-p) \gamma_{2} \frac{L^{\gamma_{2}}}{l^{\gamma_{2}+1}}, 0<p<1 . \gamma_{1}>1>\gamma_{2}$ and:

$$
\hat{K} \equiv \frac{1-\alpha}{\left[\frac{(1-p)\left(1-\gamma_{2}\right)}{p\left(\gamma_{1}-1\right)}\right]^{\frac{1}{\gamma_{1}-\gamma_{2}}}\left(\frac{R-M}{\underline{L}}\right)} \in(1, N-1)
$$

Then the individually optimal pattern of exposures $\left(a_{i j}^{*}\right)_{j \neq i}$ of any firm $i$ has $\hat{K}$ nonzero entries, all equal to

$\hat{a} \equiv\left(\frac{(1-p)\left(1-\gamma_{2}\right)}{p\left(\gamma_{1}-1\right)}\right)^{\frac{1}{\gamma_{1}-\gamma_{2}}}\left(\frac{R-M}{\underline{L}}\right)$, while the remaining $N-1-\hat{K}$ entries are all equal to 0 .

The basic intuition for the former result is as follows. If Pareto distributions with finite and 
infinite first moments are mixed, the exposure function is given by

$$
F\left(a_{i j}\right)=p a_{i j}^{\gamma_{1}}\left(\frac{\underline{L}}{R-M}\right)^{\gamma_{1}}+(1-p) a_{i j}^{\gamma_{2}}\left(\frac{\underline{L}}{R-M}\right)^{\gamma_{2}}
$$

This function is neither uniformly convex nor concave, but it changes curvature exactly once. More specifically, it turns from being concave for low levels of exposure to being convex for high levels. Naturally, the point where such change of curvature arises depends on the weights in the mixture, getting larger the smaller is the weight $p$ associated to the distribution with little mass on the tail. Under these conditions, if $p$ is low enough, the level of exposure associated to maximal risk sharing (positive and uniform exposure to all other firms) falls in the concave region of $F($.$) , hence$ bringing in the polarizing forces discussed above that make spreading exposure levels and eventually eliminating the exposure to some firms beneficial. This yields the optimality of a positive exposure level to only a subset of the firms in the system. On the other hand, when components are close to their minimal size individual exposures are larger and, provided $p$ is not too high, fall into the convex region of the function $F(\cdot)$. There, as also explained, the benefits of risk sharing prevail and push in the direction of uniform exposure towards a larger number of other firms.

The condition $\hat{K} \in(1, N-1)$ indeed ensures that $p$ is neither too low nor too high, that is both components of the distribution, one with low and the other with high mass on the tail, are attributed enough weight. Hence neither of these two polar forces at work dominates. As shown in Proposition 3, the individually optimal pattern of exposures strives to limit contagion, since positive exposure is limited to a subset of other firms, while attempting to achieve some risk sharing, with the formation of linkages to more than a single other firm. Also, the route chosen to limit contagion is only segmentation: the individually optimal component size varies continuously with the weight $p$ attributed to the two risk components, but exposure is always uniform to all firms with whom a linkage exists. It is again interesting to relate these findings to the necessary conditions stated in Proposition 1 1 the value of the positive exposure $\hat{a}$ is pinned down by the requirement $F^{\prime}(\hat{a})=F(\hat{a}) / \hat{a}$ stated in condition [3] for the same level of exposure towards a subset of other firms to be optimal and $F^{\prime}(0)>F^{\prime}(\hat{a})$, in line with condition [1]

An important feature of the environment considered in Proposition 3 is that in this case the individually optimal pattern of exposures for each firm cannot be generally implemented as a 
solution of problem $[\mathbf{S P}]$. While a component of size $\hat{K}+1$ allows to implement the individual optimum for all the firms lying in it and to satisfy the additional constraints present in subproblem $[\mathbf{P}(\hat{K})]$, the firms in the system cannot be all arranged into components that are all of that size. This would in fact require that $N$ be divisible by $\hat{K}+1$, a condition that is typically not satisfied. Hence in this situation, in order to identify the socially optimal financial structure, we must take explicitly into account the feasibility constraints that relate the exposure levels of one firm to those of other firms. How should the division of the system into components and their corresponding internal structure be adjusted so as to take such constraints into account? Is the social optimum asymmetric, with all but one components exhibiting the same size and pattern of connections as in the solution of problem $[\mathbf{I P}]$ and the last component taking the slack? Or is it rather a more symmetric configuration where all components adjust their sizes to satisfy the feasibility constraints? We provide the answer and characterize the optimum in the following result.

Proposition 4 Under the assumptions of Proposition 3, if in addition $N<2(\hat{K}+1)$, a socially optimal matrix of exposures $\left[\left(a_{i j}^{*}\right)_{j \neq i}\right]_{i=1}^{N}$ is given by one of the two following cases: either (i) two identical components of size $N / 2$ such that, in each row $i=1,2, \ldots, N$, there are $(N-2) / 2<\hat{K}$ off-diagonal elements equal to $a^{*}=(1-\alpha) 2 /(N-2)$ and the remaining ones equal to 0 , or (ii) a single component such that in each row there are multiple levels of exposure.

To gain some intuition for the result note that, if the optimum exhibited one component with the individually optimal size $\hat{K}+1$, the remaining firms should lie in one (or more) component with a smaller size, since $N<2(\hat{K}+1)$. It can then be shown that the optimal exposure levels within these two components is uniform and given respectively by $\hat{a}$ in the first one and by a value larger than $\hat{a}$ in the other, and that both values fall in the convex part of $F($.$) . Also, when K \leq \hat{K}$ the convexity of $F($.$) is inherited by the optimal value of a component V(K)$, obtained as a solution of $[\mathbf{P}(K)]$. Hence rebalancing the two components bringing their sizes closer together will reduce expected defaults in the system. A slightly modified argument yields the same result if we had one component of size bigger than $\hat{K}+1$ and one (or more) other components smaller than $\hat{K}+1$ : reducing the size of the bigger one to equal the optimal size $\hat{K}+1$ is clearly beneficial and this allows to increase the size of the smaller components which also reduces expected defaults.

Hence at the social optimum there cannot be any component of the individually optimal size 
$\hat{K}+1$, which tells us that the costs of satisfying the feasibility constraints must be spread across all firms. The optimal financial structure is either perfectly symmetric, with two identical components of size smaller than the individually optimal one and the same level of exposure within them, or is given by a single component, with firms featuring different levels of exposure to other firms. Hence it also exhibits, like the individual optimum, a combination of risk sharing and circuit-breaks to limit contagion, even if it differs in its specific form.

Next we identify a different class of environments, again in terms of probability distributions of the shocks, where the individually optimal way to limit contagion no longer entails segmentation but rather a modulation of the internal connectivity within components. To illustrate this possibility in a simpler and more transparent scenario, we consider first the case where the shock distribution is a mixture of a distribution inducing a convex risk exposure function $\bar{F}$ (.) (as e.g. in the Pareto case with low probability in the tail) and a degenerate distribution whose mass is concentrated on a single point.

Proposition 5 Suppose the distribution of $\widetilde{l}$ yields a risk exposure function of the form:

$$
F\left(a_{i j}\right)=p \bar{F}\left(a_{i j}\right)+(1-p) \mathbb{I}\left(a_{i j}>(R-M) / L_{1}\right)
$$

where $\mathbb{I}(\cdot)$ is the indicator function and $\bar{F}(\cdot)$ is strictly convex and $\bar{F}^{\prime}(0)=0$. Assume

$$
L_{1}>\frac{(R-M)(N-1)}{1-\alpha},
$$

and

$$
1-p+p\left(\bar{F}\left(\frac{1-\alpha}{N-1}\right)-\bar{F}\left(\frac{R-M}{L_{1}}\right)\right)-p \bar{F}^{\prime}\left(\frac{1-\alpha}{N-1}\right)\left(\frac{1-\alpha}{N-1}-\frac{R-M}{L_{1}}\right)>0 .
$$

Then the individually optimal pattern of exposures $\left(a_{i j}^{*}\right)_{j \neq i}$ for any firm $i$ is such that $\left\{a_{i j}^{*}\right\}_{j \neq i}=$ $\left\{a^{\prime}, a^{\prime \prime}\right\}$, for some $a^{\prime}>a^{\prime \prime}=(R-M) / L_{1}$.

The intuition for this result can be explained as follows. The convexity of $\bar{F}$ (.) and the property that $\bar{F}^{\prime}(0)=0$ implies the optimality of a positive level of exposure to all other firms in the system, that is, no segmentation. Condition (19) then states that the size $L_{1}$ of the shock occurring with 
strictly positive probability is large enough that, when a firm displays a positive and uniform level of exposure to every other firm, it always defaults when such shock hits a firm in the system. As a consequence, the only possible way to avert sure default when shock $L_{1}$ occurs is to lower the exposure to some firms below a certain threshold while increasing that to the remaining firms. In that way the firm would not default when shock $L_{1}$ hits one of the firms in the first group ${ }^{27}$ Even though this modulation of the firm's exposure levels does less well than a uniform level when a shock comes from the distribution yielding $\bar{F}($.), it is still preferable provided the probability $(1-p)$ of such a large shock is high enough, as stated in condition 20.

Next we build upon Proposition 1 and 5 to gain some more general insight regarding the conditions under which the trade-off between risk sharing and contagion is optimally resolved by lowering the dispersion of exposures within components rather than by segmentation. That is, individually optimal configurations involve a unique component and multiple levels of exposure.

Proposition 6 Let $F(\cdot)$ be twice continuously differentiable and assume that $F^{\prime}(0)=0, F^{\prime}(a)>0$ for all $a>0$. Then an individually optimal pattern of exposures $\left(a_{i j}^{*}\right)_{j \neq i}$ of any firm $i$ has the following properties: (i) $a_{i j}^{*}>0$ for every $j$, (ii) there are generically at most two distinct values $a^{\prime} \neq a^{\prime \prime}$ such that $a_{i j}^{*} \in\left\{a^{\prime}, a^{\prime \prime}\right\}$ for all $j$. Moreover, (iii) if $F^{\prime \prime}\left(\frac{1-\alpha}{N-1}\right)<0$, then $\left(a_{i j}^{*}\right)_{j \neq i}$ involves at least two distinct, strictly positive, levels of exposure.

Note that from claims (ii) and (iii) it follows that when $F^{\prime \prime}\left(\frac{1-\alpha}{N-1}\right)<0$ we have exactly two distinct strictly positive levels of exposure. Under the assumptions of Proposition 6 the function $F(\cdot)$ is convex for values of $a$ close to zero and, under the additional assumption in claim (iii), it changes curvature at least twice. Note that this change of curvature occurs in the reverse order than that contemplated in Proposition 3 and this explains the different outcome in the two cases: now the change is from convex to concave. The properties of the function $F(\cdot)$ around zero imply, as we already saw in Proposition 5 and in line with condition [1] of Proposition 1, that it is always beneficial to increase marginally the level of exposure to any other firm above zero, as stated in claim (i). From part [2] of the same proposition we know that the presence of changes in curvature

\footnotetext{
${ }^{27}$ If the distribution were truncated at $\bar{L}$, as discussed in Footnote 16 to ensure that limited liability holds, then the lowest level of exposure obtained at the solution of problem $[\mathbf{I P}]$ could alternatively be at $(R-M) / \bar{L}$ instead of at $(R-M) / L_{1}$. Such value in fact ensures the firm never defaults when any shock, not only the shocks of size $L_{1}$ or smaller, hits one of the firms towards which exposure is lower.
} 
of $F(\cdot)$ implies that the optimal solution may feature distinct but positive levels of exposure. Claims (ii) and (iii) then show that generically the optimum exhibits exactly two distinct levels.

It is then immediate to verify that in the environments considered in Propositions 5 and 6 the socially and individually optimal patterns of financial linkages coincide (provided $\varepsilon$ is not too large). The argument is the same as the one given below Proposition 2 and relies on the fact that, when the individually optimal component size is maximal, it is always possible to arrange firms in the system to generate this level of segmentation.

To sum up, we have seen that, in the presence of changes in the curvature of the risk exposure function, the optimal level of exposure among firms tends to be non-extreme and entails a combination of risk sharing and circuit-breaks to limit contagion. Depending then on the specific way in which changes in concavity occur, segmentation or the modulation of the intensity of linkages within components prove more effective in achieving that optimum. In the first case a conflict between individual and social optimum arises.

\section{Equilibrium and optimality}

The analysis undertaken in the previous section has characterized the patterns of financial exposures among firms which are optimal from a social welfare viewpoint. An important issue is whether these configurations are consistent with the choices made by individual firms - in other words, whether social and individual incentives are aligned.

To address this issue, we need to model the process of network formation among firms, specifying how individual firms decide to create or destroy links and choose their intensity, thus shaping their pattern of exposures. Such a network formation analysis can be carried out at different levels of detail, and under different assumptions on firms' behavior. Here our choice in these respects is motivated by the following two-fold objective. First, mutual exposures should require the consensus of those involved. We want then to allow firms sufficient discretion and flexibility to establish linkages with any other firm in the system. Second, we want to model the decision process regarding the intensity of linkages among firms in any connected component in a simple enough manner that the theoretical framework is rendered tractable. This suggests describing such 
internal process in a reduced form, so that only the formation of the groups of firms constituting the connected components of the system is studied from an explicitly strategic viewpoint.

To define matters formally, we first specify the network formation game and then the equilibrium concept to be applied to it. The proposed game is a variation of the classical "announcement game" used in the network literature (see Myerson (1991)) and consists of two stages:

(1) Each firm independently submits its proposals concerning the set of other firms to which it wants to connect. Formally, a strategy of each firm $i$ is then a subset $\mathcal{S}_{i} \subset \mathcal{N}$, with the convention that $i \in \mathcal{S}_{i}$.

(2) Given a profile of strategies $\mathbf{S} \equiv\left(\mathcal{S}_{i}\right)_{i \in N}$, a connected component (can also think of it as a coalition) $\mathcal{C} \subset \mathcal{N}$ is formed if, and only if, $\mathcal{S}_{i}=\mathcal{C}$ for all $i \in \mathcal{C}$. For any component $\hat{\mathcal{C}}$ that is actually formed, the pattern of linkages among the firms in $\hat{\mathcal{C}}$ is implemented that allows to minimize the probability of default of any individual firm present in the component. Note that this pattern is the same as the one obtained as the solution of problem $[\mathbf{P}(K)]$, when the number of firms in $\hat{\mathcal{C}}$ is $K+1$, and hence the probability of default of any firm in $\hat{\mathcal{C}}$ is just $V(K)$.

On the other hand if, for any firm $i, \mathcal{S}_{i}$ is such that $\mathcal{S}_{j} \neq \mathcal{S}_{i}$ for some $j \in \mathcal{S}_{i}$, $i$ 's proposal is taken to be rejected and hence firm $i$ forms no linkages (i.e. remains in isolation).

It is clear that in the network formation game described in (1)-(2) the emphasis lies on the individual decisions concerning the formation of the connected components in the system, that determine the pattern of segmentation of the system. The dispersion of the connections within each component is then chosen optimally, so as to minimize the probability of default of any firm in the component.

To study the network formation game given by (1)-(2) above, one possibility is to resort to the standard notion of Nash Equilibrium. However, an undesirable feature of such concept in this context is that it leads to a vast multiplicity of equilibrium networks. This is a consequence of the fact that the formation of any particular component requires the mutual agreement of all firms involved, which induces a coordination problem between the firms ${ }^{28}$ To address the entailed

\footnotetext{
${ }^{28}$ As an extreme illustration, note that the empty network, where each firm stays in isolation, can always be supported by a Nash equilibrium in which no firm makes a proposal to link to others.
} 
multiplicity issue, it is common in the network literature to consider a strengthening of the Nash

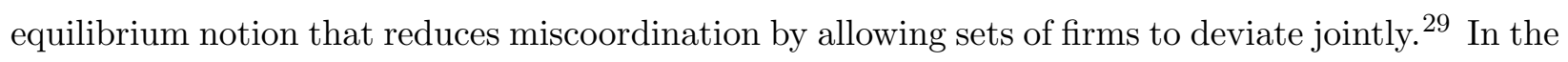
context of our model we shall introduce this possibility by means of the concept we label CoalitionProof Equilibrium (CPE), where any group (i.e. coalition) of firms can coordinate their deviations. To state it formally, for any given strategy profile $\mathbf{S}=\left(\mathcal{S}_{i}\right)_{i \in N}$ we let $\mathcal{C}_{j}(\mathbf{S})$ denote the component (induced by $\mathbf{S}$ ) to which firm $j$ belongs and, with a slight abuse of the previous notation, we write $V_{j}(\mathbf{S}) \equiv V\left(\left|\mathcal{C}_{j}(\mathbf{S})\right|\right)$ to denote the probability of default of firm $j$ in this situation, when indirectly hit by a $b$ shock (where $|\cdot|$ stands for the cardinality of the set in question).

Definition 1 A strategy profile $\mathbf{S} \equiv\left(\mathrm{S}_{i}\right)_{i \in N}$ of the network-formation game described in (1)-(2) defines a Coalition-Proof Equilibrium (CPE) if there is no subset of firms $\mathrm{W} \subseteq \mathcal{N}$ and a strategy profile for these firms, $\left(\mathcal{S}_{j}^{\prime}\right)_{j \in \mathcal{W}}$, such that

$$
\forall i \in \mathcal{W}, \quad V_{i}\left(\left(\mathcal{S}_{j}^{\prime}\right)_{j \in \mathcal{W}},\left(\mathcal{S}_{k}\right)_{k \in \mathcal{N} \backslash \mathcal{W}}\right)<V_{i}(\mathbf{S})
$$

As motivated by our previous discussion, the $C P E$ concept allows not just single firms but also arbitrary collections of them to implement jointly profitable deviations (that allow to reduce the probability of defaults of the firms deviating). This remedies the coordination problems that, as explained, render Nash equilibria an ineffective theoretical concept in this case. In essence, $C P E$ can be seen as a refinement of Nash Equilibrium that addresses the latter's vast multiplicity by postulating that it must be immune to a wider set of deviations.

The relationship between equilibria and social optima is quite straightforward for the scenarios considered in Propositions 2, 5and 6. For, as we have already explained, in those cases any socially optimal configuration that solves $[\mathbf{S P}]$ is consistent with the solutions of the individual optimization problems $[\mathbf{I P}]$ for each firm $i$. Thus the optimum can be supported as a CPE because there are no profitable deviation. Similarly, any $C P E$ outcome is a solution of $[\mathbf{S P}]$, as otherwise a profitable deviation would exist.

The above discussion suggests that, in our model, any tension between social optimality and individual incentives may only arise because of the additional constraints present in problem [SP],

\footnotetext{
${ }^{29}$ See e.g. Goyal and Vega Redondo (2007) or Calvó-Armengol and Ikiliç (2009).
} 
that relate among them the intensity of the linkages of the various firms so as to ensure the feasibility of the overall financial structure. Whenever these constraints bind, the pattern of linkages must be adjusted so that feasibility holds. Proposition 4. considers indeed a scenario where this situation arises and characterizes the socially optimal way to proceed in that case. We show in the following result that the individual response of firms, at a $C P E$, is markedly different:

Proposition 7 Under the assumptions of Proposition 4, the equilibrium matrix of exposures $\left[\left(\widetilde{a}_{i j}\right)_{j \neq i}\right]_{i=1}^{N}$ differs from the socially optimal matrix $\left[\left(a_{i j}^{*}\right)_{j \neq i}\right]_{i=1}^{N}$. Specifically, $\left[\left(\widetilde{a}_{i j}\right)_{j \neq i}\right]_{i=1}^{N}$ exhibits one component of size $\hat{K}+1$, with exposures equal to $\hat{a}$, and a second component, of lower size $N-\hat{K}-1$, with exposures equal to $a^{\prime}=(1-\alpha) /(N-\bar{K}-1)>\hat{a}$.

The core idea underlying the preceding result was already largely illustrated in the discussion following Proposition 4. There we argued that the individually optimal pattern of exposure was not a feasible outcome for all the firms in the system. While the individual aim of each firm would be to belong to a component of size $\hat{K}+1$, this objective cannot be reconciled with the inter-component symmetry required by social optimality. In a $C P E$, however, a coalition of $\hat{K}+1$ firms can form a separate component, thus leaving the residual group of firms no other choice but forming a smaller (individually suboptimal) component. This configuration, in other words, is coalitionally stable. But, as we discussed, the induced asymmetry is socially suboptimal, in the sense of producing an expected number of defaults in the whole economy that is inefficiently high. As usual, the source of the inefficiency lies in the fact that some agents do not internalize an externality. In the present case, the externality is simply that the feasibility of the financial structure imposes a precise relationship among the linkages of all individual firms both within and across components.

The existence of a conflict between efficiency and equilibrium is of course hardly novel nor surprising in the field of social networks (see e.g. Jackson and Wolinsky (1996) for an early exploration of this conflict). For, typically, the creation or destruction of any link between two agents impose externalities on others that are not internalized by the two agents involved in the linking decision 30 In our case, the nature of the externality we want to highlight is not directly related to specific links per se but rather to the size of the risk-sharing components induced by the whole array of links established by firms. To underscore this different perspective, we focus on the formation of

\footnotetext{
${ }^{30}$ In the context of risk-sharing, this tension has been studied in a recent paper by Bramoullé and Kranton (2007).
} 
components within the network. This allows us to conceive each of them as a conglomerate (or coalition) akin to, for example, a Japanese keiretsu, whose main role has been shown to be precisely that of sharing risk (Osano 1996, Dewenter 2003). Business groups in other countries have also been shown to arise for this reason (see Khanna and Yafeh 2005).

Such a coalitional perspective has also been pursued in studying the crucial problem of risksharing in the context of poor countries where formal and market-based approaches are hardly implementable. The large literature, both theoretical and empirical, that has tackled the issue has aimed to shed light on the factors that might explain why, in general, risk-sharing generally falls short of what would be (first-best) efficient - see e.g. Townsend (1994) or Fafchamps and Lund (2003). The main culprits often suggested for that state of affairs are the ones typically stressed in the economic literature: information asymmetries or/and transaction costs. The consequences, in the end, are analogous to those our analysis suggests, i.e. a distortion on the effective size of risk-sharing coalitions (see e.g. Murgai et al. (2002) for a paper that makes this point in a simple model and then tests it in a water-sharing irrigation context in Pakistan.) Our model indicates that one need not rely on these "market imperfections" to rationalize second-best arrangements of the sort described. The individual incentives underlying the coalition-formation process (which reflect only the basic trade-off between risk sharing and contagion) may be sufficient to explain the induced inefficiencies.

It is thus possible that the externality we have uncovered could be used to explain in part the size inefficiencies detected in risk sharing in developing countries, as well as the ones potentially arising from the arrangements through which business groups (and, in particular, financial institutions) in advanced economies attempt to mitigate risk. The latter inefficiencies have not been explored and the former have been attributed to other causes. A reason for this lack of empirical findings for the kind of externality we have detected might be its novel nature. Thus, we believe our paper opens a fruitful avenue for empirical research. 


\section{Heterogeneous firms}

So far we have concentrated the discussion on a situation where all firms in the system are ex ante identical. Although this allows us to obtain clear-cut analytic results and gain some understanding of the forces at play, it is important to extend our analysis to situations where, as it often happens in the real world, firms are heterogeneous, in their size or in the distribution of their shocks. This raises the novel issue for any firm of determining the type of firms with whom to establish linkages, in addition of how many linkages to form.

In this section we focus our attention on the case where the asymmetry between firms pertains to the risk properties of the shocks they face. We assume the $N$ firms are partitioned into $T$ subsets $\mathcal{N}_{1}, \ldots \mathcal{N}_{T}$, so that $\mathcal{N}=\cup_{t=1}^{T} \mathcal{N}_{t}$, and for every firm $i \in \mathcal{N}_{t}$ the $b$ shock follows a cumulative distribution function $\Phi_{t}$, for all $t \in\{1, \ldots, T\}$. The distributions $\Phi_{t}$ have the same expected value but may otherwise differ in their risk characteristics. We maintain then the assumptions that the return on all firms' projects is equal to $R$ in the absence of shocks and to $R-\underline{L}$ if a small shock hits and that the $b$ shocks hit any firm with probability $q(1-\pi)$.

Consider first the value of the expected fraction of firms who default, if indirectly hit by a shock $b$, in a homogenous component $\mathcal{C}$ with $K^{c}+1$ firms all of the same type $t$, when the intensity of linkages within the component is optimally chosen. This is simply given by the optimal value of the component obtained at a solution of problem $\left[\mathbf{P}\left(K^{c}\right)\right]$ with $F()=.1-\Phi_{t}((R-M) /$.$) , which we$ denote by $V\left(K^{c}, \Phi_{t}\right)$ for $t=1, . ., T$. On this basis we can find a lower bound for the corresponding

expression $V\left(K^{c},\left(\left|\mathcal{N}_{t}^{c}\right|, \Phi_{t}\right)_{t=1}^{T}\right)$ for a heterogeneous component, of the same size $K^{c}+1$, but now with a subset $\mathcal{N}_{t}^{c}$ of firms of type $t=1, . ., T$ :

$$
V\left(K^{c},\left(\left|\mathcal{N}_{t}^{c}\right|, \Phi_{t}\right)_{t=1}^{T}\right) \geq \sum_{t=1}^{T} \frac{\left|\mathcal{N}_{t}^{c}\right|}{K^{c}+1} V\left(K^{c}, \Phi_{t}\right) .
$$

To understand the expression on the right hand side of (21), note that $\left|\mathcal{N}_{t}^{c}\right| /\left(K^{c}+1\right)$ is the probability that, if a $b$ shock hits the component, it hits a firm of type $t$. When this happens $V\left(K^{c}, \Phi_{t}\right)$ is the expected fraction of firms indirectly hit who default, if the intensity of the linkages is optimally chosen (as in problem $\left[\mathbf{P}\left(K^{c}\right)\right]$ ) for a shock with distribution $\Phi_{t}$. This observation uses the fact that, since firms of all types have the same size, they have the same capacity for absorbing 
shocks and this implies that the expected fraction of firms defaulting only depends on the size of the component and the type $t$ of the firm hit by a $b$ shock. Hence the specific type of a firm that is only indirectly hit by a shock does not matter for its default probability. It is important to observe that the value on the right hand side of (21) is obtained by choosing optimally the intensity of linkages for each type $t$. On the other hand, since in a heterogeneous component the intensity of linkages can only take one value which cannot be adjusted according to the type $t$ of firm hit by a $b$ shocks, this expression only constitutes a lower bound for $V\left(K^{c},\left(\left|\mathcal{N}_{t}^{c}\right|, \Phi_{t}\right)_{t=1}^{T}\right)$.

We are interested in particular in identifying the optimal segmentation structure, which now requires determining not only the number of firms that should lie in each component but also - most importantly - the composition of each component in terms of the different types of firms included in it. One important concern will be to understand whether firms' matching within components should be assortative or dissortative - that is, whether components should be more or less homogeneous. In this context, therefore, a structure must specify a set of components $\{1, . ., C\}$ and, for each component $c$, the corresponding type distribution $\left\{\mathcal{N}_{t}^{c}\right\}_{t=1, \ldots, T}$, where $\mathcal{N}_{t}^{c} \subseteq \mathcal{N}_{t}$ stands for the subset of firms of type $t$ that belong to component $c$. Let $\hat{K}_{t}$ be the number of nonzero terms obtained at a solution of problem $[\mathbf{I P}]$ when the distribution of the $b$ shocks is $\Phi_{t}$ for all firms; as we saw ${ }^{31}$ in Section $2, \hat{K}_{t}$ is the value of $K$ at which the function $V\left(K, \Phi_{t}\right)$ reaches a minimal value. Thus, we have:

Proposition 8 Suppose that, for every $t$, the distribution $\Phi_{t}$ satisfies the properties stated in Proposition 3 and that the cardinality $\left|\mathcal{N}_{t}\right|$ of the set of firms of type $t$ is a multiple of $\hat{K}_{t}+1$. Then at the optimal financial structure firms belong to homogeneous components, in each of which firms are all of the same type, and $\hat{K}_{t}+1$ is the common size of every component consisting of firms of type $t$, for $t=1, . ., T$.

The intuition for this result is as follows. Conditionally on the $b$ shock hitting a firm of type $t$, the optimal arrangement is to have all components of size $\hat{K}_{t}+1$, since $\hat{K}_{t}$ is the value that minimizes $V\left(K, \Phi_{t}\right)$, and the pattern of linkages set at the values obtained at a solution of problem [IP] with $F()=.1-\Phi_{t}((R-M) /$.$) . If we had an heterogeneous component, with firms of type$ $t^{\prime}$, in addition to $t$, present in it, the size of this component - and the pattern of its connections

\footnotetext{
${ }^{31}$ See in particular the proof of Proposition 4 in the Appendix.
} 
- could not be set to equal simultaneously the (generally different) optimal values for the cases of shocks hitting type $t$ and type $t^{\prime}$ firms. Hence the expected number of defaults could be reduced by rearranging firms within homogeneous components, as this would allow to attain the minimum of $V\left(K, \Phi_{t}\right)$ for each $t$ (and, under the stated assumptions, this is always feasible).

The result says that, whenever some segmentation is beneficial, the optimal financial structure exhibits perfectly assortative matching, that is safer firms will only establish linkages with firms with an analogous risk profile and belong to large components, while riskier firms have only exposure to riskier firms and lie in smaller components. Of course, a key feature required for this conclusion to hold is that, as already noticed, the expected number of indirect defaults in a component only depends on the type $t$ of the firm directly hit by the shock and the size of the component.

When instead firms are heterogeneous in their size, that is in the level of their resources and the likelihood of shocks hitting them, the conclusion is somewhat different. As we show in the Appendix an irrelevance result holds in that case: once the exposure of any firm to large and small firms is suitably modulated to "offset" the effect of size, heterogeneous components feature the same default probability as homogeneous ones. Hence the presence of any other, even small, advantage for small firms to connect to large firms, based on different considerations than risk sharing (for instance informational processing reasons if information acquisition has increasing returns to scale), would lead to the formation of components where large and small firms coexist, as in the case of core periphery networks.

\section{Conclusion}

We have proposed a stylized model to study the problem that arises when firms establish financial linkages among them to weather shocks that can threaten their survival, but by so doing they become exposed to the risk coming from those same connections. We have then characterized the patterns of linkages among firms that allow to minimize default in the system for different properties of the distribution of the shocks that may hit firms. For distributions that exhibit a property we identify as uniform curvature of the risk exposure function, the optimum is at one of two extremes: either full integration or maximal segmentation. On the other hand, when this condition does not 
hold, we find that the use of intermediate degrees of segmentation or the modulation of the levels of exposure to other firms allow to strike an optimal balance between limiting contagion while still allowing some risk sharing. We also show that which of these two instruments proves more effective depends on the specific way in which changes in curvature occur.

We have also explored whether such social optimum is aligned with the choices of individual firms to establish linkages with other firms. There is a conflict between individual incentives and social optima, due to the fact that firms have always an incentive to form connected components of the size that minimizes the default probability of their members. By so doing, they ignore the negative externality this behavior sometimes imposes on other firms because of the feasibility constraints that relate the admissible linkages of one firm to those of other firms ${ }^{32}$ Finally, we have found that when we allow for heterogeneity among firms in the risk properties of the shocks they receive, optimality requires perfect assortativity, that is to have homogeneous components, in each of which the risk characteristics of the shocks affecting firms is the same.

The basic insights obtained from this analysis can inform the regulation and design of financial systems in the real world. Should one separate commercial and investment banking? Should the financial systems of different regions be insulated from each other, or should overall integration be pursued? And, in the end, how effectively can we trust any prescription along these lines to be indeed implemented by banks (i.e. to be compatible with their own individual incentives)? These are some of the crucial questions that naturally arise, and to which we have shown our model can provide answers based on the analysis of some key factors at play.

Loepfe et al. (2013) extends our analysis through numerical methods to environments with more realistic features and verifies the robustness of our main results. More precisely, it finds that the optimality of forming smaller/less dense networks when shocks come from distributions that put more weight on large shocks extend to the cases where we allow for the possibility of shocks hitting multiple firms at the same time and for networks where the balance condition (4) may not hold. In a different vein, they also consider the effects of shocks to the real-life network of corporate

\footnotetext{
${ }^{32} \mathrm{~A}$ further conflict between social and individual incentives could arise when welfare is assessed in terms of a convex - rather than linear - function of the number of defaults in the system, as briefly discussed in footnote 21 . This would create a wedge between the objective pursued in the social optimum problem and in the individual firm problem, since the firm naturally cares only about its own survival. As a consequence the social optimum would generally diverge from the individual one, typically by having lower and less dispersed exposures.
} 
control studied by Battiston et al. (2012) and find that they depend in important ways on the nature of the shock distribution as suggested by our theory.

But, of course, a proper discussion of the risk-sharing and contagion phenomena in the real world must also account for many important dimensions from which our model abstracts. One of them is the consideration of problems of moral hazard and, in general, asymmetries of information that have been repeatedly singled out as a key factor underlying the recent financial crisis. We also ignore forces - intermediation, for instance, or also cooperation and the exploitation of synergies that, beyond risk-sharing and limiting contagion, contribute to determining the form of financial networks. A proper study of all these crucial issues will demand a richer theoretical framework and a more powerful methodology, the development of which can hopefully build in a fruitful manner upon the present work.

\section{Appendix}

\section{Proof of Proposition 1}

To establish part [1], let $\lambda$ be the Lagrange multiplier of constraint 12 in problem [IP]. Suppose $a_{i j}^{*}=0$. Then from $(12)$ it follows that we must have $a_{i k}^{*}>0$ for some $k \neq i$. From the first order conditions in problem $[\mathbf{I P}]$ it then follows that

$$
F^{\prime}(0)=F\left(a_{i j}^{*}\right) \geq F^{\prime}\left(a_{i k}^{*}\right)=\lambda .
$$

Similarly, to prove part [2] note that, if $a_{i j_{l}}^{*} \neq a_{i j_{m}}^{*}$ belong to a solution of [IP], the first order conditions of this problem imply: $F^{\prime}\left(a_{i j_{l}}^{*}\right)=F^{\prime}\left(a_{i j_{m}}^{*}\right)$. Hence $F^{\prime \prime}(a)$ cannot have the same sign for all $a_{i j} \in\left(a_{i j_{l}}^{*}, a_{i j_{m}}^{*}\right)$, which implies the function needs to change convexity between $a_{i j_{l}}^{*}$ and $a_{i j_{m}}^{*}$. Since there are $p$ distinct elements with this property, this implies that convexity changes at least $p-1$ times.

To establish part [3], suppose the solution is characterized by $k \geq 2$ distinct values $\bar{a}_{t}, t \in$ 
$\{1, \ldots, k\}$, each of them repeated $n_{t}>0$ times. Then $n_{t}, \bar{a}_{t}$ for $t \in\{1, \ldots, k\}$ must solve the problem

$$
\begin{aligned}
\min _{\left(n_{t}, \bar{a}_{t}\right)_{t=1}^{k}} \sum_{t=1}^{k} n_{i} F\left(\bar{a}_{t}\right) \\
\text { s.t. } \sum_{t=1}^{k} n_{t} \bar{a}_{t}=1-\alpha, \\
\sum_{t=1}^{k} n_{t}=N-1 \\
1-\alpha \geq \bar{a}_{t} \geq 0 ; n_{t} \geq 0
\end{aligned}
$$

Suppose first that $\bar{a}_{v}=0$ for some $v \in\{1, \ldots, k\}$. Then at the first order conditions of the above problem we must have

$$
F\left(\bar{a}_{v}\right)=F(0)=\mu
$$

Since $F(0)=0$, this implies that $\mu=0$. For $t \in\{1, \ldots, k\}, t \neq v$, we have then $\bar{a}_{t}>0$ and

$$
\begin{aligned}
F\left(\bar{a}_{t}\right) & =\lambda \bar{a}_{t}+\mu, \\
n_{t} F^{\prime}\left(\bar{a}_{t}\right) & =\lambda n_{t}
\end{aligned}
$$

Using the fact that $\mu=0$, we obtain

$$
F^{\prime}\left(\bar{a}_{t}\right)=\frac{F\left(\bar{a}_{t}\right)}{\bar{a}_{t}}
$$

If, instead $\bar{a}_{t}>0$ for all $t \in\{1, \ldots, k\}$, then $(24)$ must hold for all $t$ and hence:

$$
F\left(\bar{a}_{t}\right)=F^{\prime}\left(\bar{a}_{t}\right) \bar{a}_{t}+\mu
$$

\footnotetext{
${ }^{33}$ As already noticed in the text, the constraint that $n_{t}$ is an integer, for each $t=1, . ., k$, is ignored here for simplicity.
} 
Subtracting the expression for $\bar{a}_{t}$ from that for $\bar{a}_{v}$ yields

$$
F\left(\bar{a}_{t}\right)-F^{\prime}\left(\bar{a}_{t}\right) \bar{a}_{t}=F\left(\bar{a}_{v}\right)-F^{\prime}\left(\bar{a}_{v}\right) \bar{a}_{v}
$$

Using then the fact that, by $[2], F^{\prime}\left(\bar{a}_{t}\right)=F^{\prime}\left(\bar{a}_{v}\right)$ we readily obtain the claimed property. $\square$

\section{Proof of Remark 2.}

From (9) we obtain

$$
F^{\prime \prime}\left(a_{i j}\right)=-\frac{(R-M)^{2}}{a_{i j}^{4}} \Phi^{\prime \prime}\left(\frac{R-M}{a_{i j}}\right)-2 \frac{(R-M)}{a_{i j}^{3}} \Phi^{\prime}\left(\frac{R-M}{a_{i j}}\right) \geq 0
$$

hence

$$
-\frac{R-M}{a_{i j}} \Phi^{\prime \prime}\left(\frac{R-M}{a_{i j}}\right)-2 \Phi^{\prime}\left(\frac{R-M}{a_{i j}}\right) \geq 0,
$$

from which the stated property readily obtains. Also, the log concavity of $\Phi($.$) requires$

$$
\frac{\partial^{2}(\log \Phi(x))}{\partial x^{2}}=\frac{\Phi^{\prime \prime}(x) \Phi(x)-\left(\Phi^{\prime}(x)\right)^{2}}{\Phi^{2}(x)}<0,
$$

or

$$
\frac{\Phi^{\prime \prime}(x)}{\Phi^{\prime}(x)}<\frac{\Phi^{\prime}(x)}{\Phi(x)}
$$

always satisfied if (26) holds. A symmetric argument establishes the other property.

\section{Proof of Proposition 2}

Let us consider problem $[\mathbf{I P}]$. Note that this problem always has a solution. Assume first that $F($.$) is convex and suppose that, at a solution of [\mathbf{I P}]$ we have $a_{i j} \neq a_{i k}$ for $j, k \neq i$. By the convexity of $F(\cdot)$ we have:

$$
2 F\left(\frac{a_{i j}+a_{i k}}{2}\right)<F\left(a_{i j}\right)+F\left(a_{i k}\right) .
$$

Hence replacing both $a_{i j}$ and $a_{i k}$ with $\left(a_{i j}+a_{i k}\right) / 2$ still satisfies the constraints of problem [IP] and yields a strictly lower value of the objective function, thus a contradiction. It then follows that at a solution of $[\mathbf{I P}]$ we must have $a_{i j}=a_{i k}$ for all $j, k \neq i$ and hence $a_{i j}=\frac{1}{2(N-1)} \quad \forall j \neq i$.

Assume next that $F($.$) is concave and suppose that, at a solution of problem [\mathbf{I P}] a_{i j} \neq 0$, 
$a_{i k} \neq 0$ for some $j, k \neq i$. By the concavity of $F(\cdot)$ we get:

$$
\begin{aligned}
& \frac{a_{i j}}{a_{i j}+a_{i k}} F\left(a_{i j}+a_{i k}\right)+\frac{a_{i k}}{a_{i j}+a_{i k}} F(0)<F\left(a_{i j}\right), \\
& \frac{a_{i k}}{a_{i j}+a_{i k}} F\left(a_{i j}+a_{i k}\right)+\frac{a_{i j}}{a_{i j}+a_{i k}} F(0)<F\left(a_{i k}\right) .
\end{aligned}
$$

Summing the two inequalities yields:

$$
F\left(a_{i j}+a_{i k}\right)+F(0)<F\left(a_{i j}\right)+F\left(a_{i k}\right)
$$

hence replacing $a_{i j}, a_{i k}$ with the pair $\left(a_{i j}+a_{i k}\right), 0$ is still a feasible configuration for problem [IP] and allows to lower the value of the objective function, again a contradiction. Thus at a solution of $[\mathbf{I P}]$ we can only have one nonzero off diagonal entry of $A$, that is $a_{i j}=1-\alpha$ for some $j \neq i$.

Consider then a matrix $A$ where the elements of each row are equal to a solution of $[\mathbf{I P}]$ for each $i=1, . ., N$. In the first case we get a matrix with all the off-diagonal elements strictly positive and equal to $(1-\alpha) /(N-1)$, that is a single component with uniform exposure. This matrix clearly satisfies the additional constraints present in subproblem $[\mathbf{P}(\mathrm{N}-1)]$ : the balance condition (4) and the condition that, within the component, every firm is linked to every other firm lying in it 34 and so is also a solution of $[\mathbf{S P}]$. When instead $F($.$) is concave the individually optimal component size,$ given by the number of strictly positive entries at a solution of $[\mathbf{I P}]$ is 2 . It is then immediate to see that the matrix $A_{K}$ with off-diagonal elements equal to $(1-\alpha) / K$ and $K=1$ implements the solution of $[\mathbf{I P}]$ for any pair of distinct values of $i$ and satisfies the additional constraints present in subproblem $[\mathbf{P}(1)]$. Moreover, since $N$ is assumed to be even, the firms in the system can always be arranged so as to implement the individually optimal component size (2 in this case), for every component. Hence this specification also solves problem $[\mathbf{S P}]$.

\section{Proof of Proposition 3}

Differentiating the function $F\left(a_{i j}\right)$ in 17$)$ with respect to $a_{i j}$ we obtain the following expression

\footnotetext{
${ }^{34}$ Strictly speaking, this is true provided $\varepsilon \leq(1-\alpha) /(N-1)$, a natural requirement on admissible financial structures.
} 
for the derivative of $F($.$) :$

$$
F^{\prime}\left(a_{i j}\right)=p \gamma_{1} a_{i j}^{\gamma_{1}-1}\left(\frac{\underline{L}}{R-M}\right)^{\gamma_{1}}+(1-p) \gamma_{2} a_{i j}^{\gamma_{2}-1}\left(\frac{\underline{L}}{R-M}\right)^{\gamma_{2}}
$$

Since $\gamma_{1}>1$ and $\gamma_{2}<1$ the inequality

$$
F^{\prime \prime}\left(a_{i j}\right)=p \gamma_{1}\left(\gamma_{1}-1\right) a_{i j}^{\gamma_{1}-2}\left(\frac{\underline{L}}{R-M}\right)^{\gamma_{1}}+(1-p) \gamma_{2}\left(\gamma_{2}-1\right) a_{i j}^{\gamma_{2}-2}\left(\frac{\underline{L}}{R-M}\right)^{\gamma_{2}}>0
$$

holds if and only if

$$
a_{i j}>a^{* *} \equiv\left(\frac{(1-p) \gamma_{2}\left(1-\gamma_{2}\right)}{p \gamma_{1}\left(\gamma_{1}-1\right)}\right)^{\frac{1}{\gamma_{1}-\gamma_{2}}}\left(\frac{R-M}{\underline{L}}\right) .
$$

Thus the function $F^{\prime}\left(a_{i j}\right)$ is first decreasing and then increasing.

We first establish that with this specification of $F($.$) , in the solution of the [IP] problem$ there cannot be $k \geq 2$ distinct values $\bar{a}_{t}, t \in\{1, \ldots, k\}$, each of them repeated $n_{t}>0$ times. To establish this, we proceed by contradiction, using again the simplified problem (22) in the Proof of Proposition 1. By part (ii) of Proposition 1 we know that $k=2$, as $F$ (.) changes concavity only once, so that we can only have two distinct values $\bar{a}_{1}>0$ and $\bar{a}_{2}>0$ each of them repeated $n_{1}$ and $n_{2}$ times respectively. Furthermore one of them, say $\bar{a}_{1}<a^{* *}$, and $\bar{a}_{2}>a^{* *}$. Thus $F^{\prime \prime}\left(\bar{a}_{1}\right)<0$. The concavity of $F($.$) at \bar{a}_{1}$ implies that for $\delta$ small $\frac{n_{1}}{2} F\left(\bar{a}_{1}+\delta\right)+\frac{n_{1}}{2} F\left(\bar{a}_{1}-\delta\right)<n_{1} F\left(\bar{a}_{1}\right)$, which establishes that the default probability can be reduced by moving away from the proposed solution, thus establishing a contradiction.

The previous argument shows that there can only be one positive value of $a>0$ in the solution of problem (22) so that we can rewrite the problem (noting that $F(0)=0$ ) as

$$
\begin{aligned}
& \min _{n, a} n F(a) \\
\text { s.t. } n a= & 1-\alpha, \\
1 \leq & n \leq N-1
\end{aligned}
$$

The first order conditions for an interior solution of such problem are

$$
F^{\prime}(a)=\frac{F(a)}{a}
$$


The solution of this equation is then uniquely given by ${ }^{35}$.

$$
a^{*}=\left(\frac{(1-p)\left(1-\gamma_{2}\right)}{p\left(\gamma_{1}-1\right)}\right)^{\frac{1}{\gamma_{1}-\gamma_{2}}}\left(\frac{R-M}{\underline{L}}\right)
$$

and satisfies the constraints of problem $(29)$ since, under the condition stated in the proposition, we have $n^{*} \in(1, N-1)$. In addition, note that $\frac{1-\alpha}{a} F(a)$ is decreasing for $a<a^{*}$ and increasing for $a>a^{*}$, thus $\left(a^{*}, n^{*}\right)$ is indeed a solution of problem 29$]$.

\section{Proof of Proposition 4}

To characterize the solution of problem $[\mathbf{S P}]$ in the case under consideration, it is convenient to consider the following constrained version of problem $[\mathbf{I P}]$, where the number of off-diagonal elements that could be positive is constrained to be $\bar{K}$, with $1 \leq \bar{K}<N-1$ :

$$
\begin{aligned}
{[\mathbf{I P}(\overline{\mathbf{K}})] \quad \min _{\left(a_{i j}\right)_{j \neq i}} \sum_{j \neq i}^{\bar{K}+1} F\left(a_{i j}\right) } & \text { s. t. } \\
\sum_{j \neq i}^{\bar{K}+1} a_{i j} & =1-\alpha \\
a_{i j} & \geq 0, \quad \forall j \neq i
\end{aligned}
$$

Let $D(\bar{K})$ denote the value of $\sum_{j \neq i} F\left(a_{i j}\right)$ at a configuration that solves $[\mathbf{I P}(\bar{K})]$, for some given $\bar{K}$.

Applying again the same method followed in analyzing problem $[\mathbf{I P}]$ in the proof of Proposition 3. we can show that in the solution of $[\mathbf{I P}(\bar{K})]$ there cannot be $k \geq 2$ distinct values $\bar{a}_{t}, t \in\{1, \ldots, k\}$, each of them repeated $n_{t}>0$ times. Thus, the solution is obtained from the following simplified

\footnotetext{
${ }^{35}$ Substituting the expression of $F(\cdot)$ in the equation $a=F(a) / F^{\prime}(a)$ we obtain in fact:

$$
p \gamma_{1} a^{\gamma_{1}-1}\left(\frac{\underline{L}}{R-M}\right)^{\gamma_{1}}+(1-p) \gamma_{2} a^{\gamma_{2}-1}\left(\frac{\underline{L}}{R-M}\right)^{\gamma_{2}}=\frac{p a^{\gamma_{1}}\left(\frac{\underline{L}}{R-M}\right)^{\gamma_{1}}+(1-p) a^{\gamma_{2}}\left(\frac{\underline{L}}{R-M}\right)^{\gamma_{2}}}{a}
$$
}

which has a unique solution for $a$ given by the expression in 30 . 
constrained problem:

$$
\begin{aligned}
& D(\bar{K}) \equiv \min _{K} K F\left(\frac{1-\alpha}{K}\right) \\
& \text { s.t. } K \leq \bar{K}
\end{aligned}
$$

Evidently, for all $\bar{K} \geq \hat{K}$ the constraint $K \leq \bar{K}$ is not binding and the optimum is the same as the one characterized in the previous proof, that is $D(\bar{K})=\hat{K} F((1-\alpha) / \hat{K})$. Clearly for $\bar{K}<\hat{K}$ the solution obtains when $D(\bar{K})=\bar{K} F((1-\alpha) / \bar{K})$, since $K F\left(\frac{1-\alpha}{K}\right)$ is decreasing for $K<\hat{K}$.

It is immediate to see that, when $\bar{K} \leq \hat{K}$, the solution of problem (31), replicated for each row $i=1, . ., \bar{K}+1$ of the matrix $A_{\bar{K}}$ also constitutes a solution of problem $[\mathbf{P}(\bar{K})]$ since the additional constraints present in the latter problem are satisfied at this solution. Hence we have $V(\bar{K})=D(\bar{K})$. In contrast, for $\bar{K}>\hat{K}$ the solution of $(31)$, replicated for $i=1, . ., \bar{K}+1$, violates the last constraint of problem $[\mathbf{P}(\bar{K})]$ and hence in that case we have $V(\bar{K}) \geq D(\bar{K})$.

We turn then our attention to problem $[\mathbf{S P}]$. Having assumed that $N<2(\hat{K}+1)$ we cannot have components that are all of size $\hat{K}+1$. We show in what follows that if the social optimum features more than one component, then they have to be of the same size, $N / 2<\hat{K}+1$. We cannot in fact have a component of size $K_{i}>\hat{K}$ and another one of size $K_{j}<\hat{K}$ at a solution of [SP]. To see that, note that $K_{i}-\hat{K}<\hat{K}-K_{j}$ since $K_{i}+K_{j}=N-2<2 \hat{K}$. Hence we can always reduce $K_{i}$ to be equal to $\hat{K}$ and increase $K_{j}$ to $K_{j}+K_{i}-\hat{K}<\hat{K}$ and since $V(\hat{K})<V\left(K_{i}\right)$ and $V\left(K_{j}+K_{i}-\hat{K}\right)<V\left(K_{j}\right)$ since $V($.$) is strictly decreasing for K<\hat{K}$.

Also, we cannot have a component of size $K_{i}<\hat{K}$ and another one of size $K_{j} \leq \hat{K}$ with $K_{i} \neq K_{j}$, by the convexity of $V($.$) for \bar{K} \leq \hat{K}$. We have in fact

$$
\begin{aligned}
V^{\prime \prime}(\bar{K}) & =-F^{\prime}((1-\alpha) / \bar{K})(1-\alpha) / \bar{K}^{2}+F^{\prime}((1-\alpha) / \bar{K})(1-\alpha) / \bar{K}^{2}+F^{\prime \prime}((1-\alpha) / \bar{K})(1-\alpha)^{2} / \bar{K}^{2} \\
& =F^{\prime \prime}((1-\alpha) / \bar{K})(1-\alpha)^{2} / \bar{K}^{2}>0
\end{aligned}
$$

by the convexity of $F(\cdot)$ at $\bar{a}>\hat{a}$. Given the assumption that $N<2(\hat{K}+1)$ we cannot have two components both of size bigger than $\hat{K}$. We are then only left with the possibility of having components all of the same size $K^{*}<\hat{K}$.

The above argument shows that expected defaults in the system are lower with two equal sized 
components that in any other configuration with two or more components. It remains however to consider the possibility of an arrangement with a single component of size $N$. It is immediate to see that in the solution to problem $[\mathbf{P}(N-1)]$ the constraint (11) must be binding, since otherwise one could simply replicate the solution to problem $[\mathbf{I P}]$ with a suitable rearrangement of elements in each row to satisfy the balance condition 10 .

\section{Proof of Proposition 5}

Under the conditions stated in the proposition, the objective function of problem $[\mathbf{I P}]$ takes the following form:

$$
\sum_{j \neq i}\left(p \bar{F}\left(a_{i j}\right)+(1-p) \mathbb{I}\left(a_{i j}>(R-M) / L_{1}\right)\right)
$$

where recall that $\mathbb{I}(\cdot)$ denotes the indicator function. First, note that at a solution of $[\mathbf{I P}]$ we cannot have two values $a_{i j} \neq a_{i k}$ with $a_{i j} \leq(R-M) / L_{1}, a_{i k} \leq(R-M) / L_{1}$. This is immediate because otherwise we can always find an improvement: the choice $a_{i j}^{\prime}=a_{i k}^{\prime}=\left(a_{i j}+a_{i k}\right) / 2$ is in fact feasible, does not affect the value of the second part of the objective function in $(32)$, and by the convexity of $\bar{F}($.$) decreases the value of the first part, where \bar{F}($.$) appears. By a symmetric$ argument it also follows that there cannot be two values $a_{i j} \neq a_{i k}$ with $a_{i j}>(R-M) / L_{1}$, $a_{i k}>(R-M) / L_{1}$.

Condition 190 implies that we must have ${ }^{36} a_{i k}>(R-M) / L_{1}$ for at least some $k \neq i$. It then follows that there can be no value $a_{i j}<(R-M) / L_{1}$. If $a_{i j}<(R-M) / L_{1}$, the alternative choice $a_{i j}^{\prime}=(R-M) / L_{1}$ and $a_{i k}^{\prime}=a_{i k}-\left((R-M) / L_{1}-a_{i j}\right)$ would allow an improvement: it is feasible, does not affect the second part of the objective function and by convexity of $\bar{F}($.) decreases the first part. Hence there can be no term $a_{i j}=0$, proving there has to be a single component.

From the above arguments it follows that at a solution of $[\mathbf{I P}]$ we have $N-H-1 \geq 0$ terms with exposure $a_{i j}=(R-M) / L_{1}$ and the remaining $H \geq 0$ terms with equal exposure $a>(R-M) / L_{1}$, such that

$$
(N-H-1) \frac{R-M}{L_{1}}+H a=1-\alpha .
$$

\footnotetext{
${ }^{36}$ If $a_{i j} \leq(R-M) / L_{1}$ for all $j$ feasibility is violated.
} 
Solving this equation for $a$ in terms of $H$ and substituting into the objective function, we obtain that the optimal value of $H$ solves the following problem 37 .

$$
\min _{N-1 \geq H} H(1-p)+p H \bar{F}\left(\frac{1}{H}\left(1-\alpha-(R-M) \frac{N-H-1}{L_{1}}\right)\right)+p(n-H-1) \bar{F}\left(\frac{R-M}{L_{1}}\right)
$$

The first-order condition for an interior solution is

$$
(1-p)+p \bar{F}(a)+p \bar{F}^{\prime}(a)\left(-a+\frac{R-M}{L_{1}}\right)-p \bar{F}\left(\frac{R-M}{L_{1}}\right)=0
$$

while the condition for a corner solution at $H=N-1$ is:

$$
(1-p)+p\left(\bar{F}\left(\frac{1-\alpha}{N-1}\right)-\bar{F}\left(\frac{R-M}{L_{1}}\right)\right)+p \bar{F}^{\prime}\left(\frac{1-\alpha}{N-1}\right)\left(-\frac{1-\alpha}{N-1}+\frac{R-M}{L_{1}}\right) \leq 0 .
$$

Under condition (20) the latter inequality never holds, hence the solution is interior, with exposure $(R-M) / L_{1}$ to a positive number of, but not all, other firms, and higher exposure $a$ to the remaining firms.

Since the solution we obtained features a single component, by a similar argument to that in the proof of Proposition 2, it satisfies the additional constraints present in problem $[\mathbf{S P}],[10$ and (11) (provided $\varepsilon$ is not too large), and hence it also solves problem $[\mathbf{S P}]$.

\section{Proof of Proposition 6}

From part [1] of Proposition 1 and the stated assumptions that $F^{\prime}(0)=0$ and $F^{\prime}(a)>0$ for all $a>0$, it immediately follows that $a_{i j}^{*}>0$ for every $j$ which establishes claim (i).

Next we establish claim (ii) by contradiction. Suppose that there were three distinct values $a^{\prime} \neq a^{\prime \prime} \neq a^{\prime \prime \prime}$, such that there exists $j_{k_{1}}, j_{k_{2}}$ and $j_{k_{3}}$ such that $a_{i j_{k_{1}}}^{*}=a^{\prime}, a_{i j_{k_{2}}}^{*}=a^{\prime \prime}, a_{i j_{k_{3} .}}^{*}=a^{\prime \prime \prime}$. Then it must be the case, by part [3] of Proposition 1 that

$$
\frac{F\left(a^{\prime}\right)-F\left(a^{\prime \prime}\right)}{a^{\prime}-a^{\prime \prime}}=\frac{F\left(a^{\prime}\right)-F\left(a^{\prime \prime \prime}\right)}{a^{\prime}-a^{\prime \prime \prime}}=F^{\prime}\left(a^{\prime}\right)=F^{\prime}\left(a^{\prime \prime}\right)=F^{\prime}\left(a^{\prime \prime \prime}\right) .
$$

\footnotetext{
${ }^{37}$ Note that at a solution of 33 we must have $H>0$, hence the non-negativity constraint on this variable never binds and can be ignored.
} 
These are four equations in three unknowns, thus a small perturbation of the function $F($.$) implies$ by standard arguments that the above systems has generically no solutions.

Finally, to establish claim (iii) we proceed by contradiction. Suppose there is a single strictly positive level of exposure. By claim (i) this has to be at $a=\frac{1-\alpha}{N-1}$, since $a_{i j}^{*}>0$ for every

$j$. If $F^{\prime \prime}\left(\frac{1-\alpha}{N-1}\right)<0$ the concavity of $F($.$) at \frac{1-\alpha}{N-1}$ implies that for $\delta$ small $\frac{N-1}{2} F\left(\frac{1-\alpha}{N-1}+\delta\right)+$ $\frac{N-1}{2} F\left(\frac{1-\alpha}{N-1}-\delta\right)<(N-1) F\left(\frac{1-\alpha}{N-1}\right)$ which establishes that the default probability can be reduced by moving away from the single level of exposure to two distinct ones, thus establishing a contradiction.

\section{Proof of Proposition 7}

We show first that for each firm $j$ the size of the component $\mathcal{C}_{j}(\mathbf{S})$ to which $j$ belongs has size $\left|\mathcal{C}_{j}(\mathbf{S})\right|+1 \leq \hat{K}+1$, where $\hat{K}$ is the value obtained at the solution of the individual problem [IP], characterized in Proposition 3. That is, no component in the CPE has size larger or equal to the individually optimal one. Suppose not: $\left|\mathcal{C}_{j}(\mathbf{S})\right|>\hat{K}$ for some $j$. A subset $\mathcal{W} \subset \mathcal{C}_{j}(\mathbf{S})$ formed of $\hat{K}+1$ firms can then profitably deviate by severing the linkages with the other firms in $\mathcal{C}_{j}(\mathbf{S})$ and attain a lower probability of default, since $V(K)$ attains indeed a minimum at $\hat{K}$.

Next, we show that at a $C P E$ we have $\left|\mathcal{C}_{j}(\mathbf{S})\right|<\hat{K}$ for some $j$ and $\left|\mathcal{C}_{i}(\mathbf{S})\right|=\hat{K}$ for all other $\mathcal{C}_{i}(\mathbf{S}) \neq \mathcal{C}_{j}(\mathbf{S})$. We proceed again by contradiction. Suppose there were two distinct components $\mathcal{C}_{i}(\mathbf{S}), \mathcal{C}_{j}(\mathbf{S})$ such that $0<\left|\mathcal{C}_{j}(\mathbf{S})\right| \leq\left|\mathcal{C}_{i}(\mathbf{S})\right|<\hat{K}$. Pick then a subset of firms belonging to the first component $\mathcal{W}^{\prime} \subset \mathcal{C}_{i}(\mathbf{S})$ so that the set given by the union of $\mathcal{W}^{\prime}$ and $\mathcal{C}_{j}(\mathbf{S})$ has $\hat{K}+1$ firms in it. The firms in $\mathcal{W}^{\prime} \cup \mathcal{C}_{j}(\mathbf{S})$ can deviate by severing the linkages of the firms in $\mathcal{W}^{\prime}$ with the other firms in $\mathcal{C}_{i}(\mathbf{S}) \backslash \mathcal{W}^{\prime}$ and forming new linkages with all the firms in $\mathcal{C}_{j}(\mathbf{S})$. By so doing each firm in $\mathcal{W}^{\prime} \cup \mathcal{C}_{j}(\mathbf{S})$ attains a lower level of its probability of default, equal to $V(\hat{K})$. Hence all firms involved benefit from the deviation, a contradiction. This establishes the above claim and completes so the proof of the Proposition.

\section{Proof of Proposition 8}

Suppose the system is arranged into $C$ components of size $K^{c}+1$, for $c=1, \ldots, C$, and in each component $c$ there are $\left|\mathcal{N}_{t}^{c}\right|$ firms of type $t$. Using (21), the expected number of firms in the system that default when indirectly hit by a $b$ shocks (and when the linkages within components 
are optimally chosen) is then

$$
\sum_{c=1}^{C}\left(K^{c}+1\right) V\left(K^{c},\left(\left|\mathcal{N}_{t}^{c}\right|, \Phi_{t}\right)_{t=1}^{T}\right) \geq \sum_{c=1}^{C}\left(K^{c}+1\right) \sum_{t=1}^{T} \frac{\left|\mathcal{N}_{t}^{c}\right|}{K^{c}+1} V\left(K^{c}, \Phi_{t}\right) .
$$

Exchanging the summation indices, the term on the right hand side of the above inequality satisfies the following

$$
\begin{aligned}
\sum_{c=1}^{C} \sum_{t=1}^{T}\left|\mathcal{N}_{t}^{c}\right| V\left(K^{c}, \Phi_{t}\right) & \geq \sum_{t=1}^{T} \sum_{c=1}^{C}\left|\mathcal{N}_{t}^{c}\right| V\left(\hat{K}_{t}, \Phi_{t}\right) \\
& =\sum_{t=1}^{T}\left|\mathcal{N}_{t}\right| V\left(\hat{K}_{t}, \Phi_{t}\right)
\end{aligned}
$$

Note that, since for each $t\left|\mathcal{N}_{t}\right|$ is assumed to be a multiple of $\hat{K}_{t}+1, \sum_{t=1}^{T}\left|\mathcal{N}_{t}\right| V\left(\hat{K}_{t}, \Phi_{t}\right)$ is also the expected number of defaults in the system if every firm of type $t$ is part of a component with $\hat{K}_{t}$ other firms all of the same type $t$ (with the pattern of linkages set at the values obtained from a solution of problem $[\mathbf{I P}]$ when the distribution of the $b$ shocks is $\Phi_{t}$ for all firms). The above inequality shows that a segmentation of the system into homogenous components allows to attain a lower number of expected defaults than any other pattern of segmentation. Moreover, as long as the value of $\hat{K}_{t}$ and/or the optimal intensity of linkages in a component vary non-trivially with the firms' type $t$, the above inequalities are strict, hence all optimal financial structures exhibit segmentation into homogenous components.

Firms heterogeneous in size For simplicity, we consider a situation with just two possible sizes. On the one hand, there are firms of unit size, identical to the ones we have been considering so far. On the other hand, there are firms of size $\beta>1$, with such larger size having the following two implications. First, the return on the projects of these larger firms when no shock hits them is $\beta R$, i.e. it is scaled up by $\beta$ compared to that of the smaller firms (naturally the same factor $\beta$ applies to the value of these firms' liabilities, which are then $\beta M$ ). Second, the larger firms face a probability of being directly hit by a shock that is $\beta$ times larger (that is, equal to $\beta q$ ). Thus the present scenario, where size affects the probability of arrival of the shocks, not their distribution, can be viewed as complementary to the one considered in Section 4 . Also, here one can view a 
large firm as a merger of $\beta$ unit-sized firms.

Of course, condition (4), ensuring some balance between the exposure of a firm to other firms, and from the other firms to this firm, needs to be properly reformulated when we consider exposures between firms of different size. The level of the exposure of a firm of size 1 to a large firm (of size $\beta$ ) should 'count' $\beta$ times an exposure of the same level to a firm of unit size (because of the higher probability of a shock). Vice versa, for a large firm the exposure to a smaller (unit size) firm counts as $1 / \beta$ times the exposure to a firm of the same size. Let us denote by $\mathcal{N}_{1}$ the subset of firms of size 1 , and by $\mathcal{N}_{\beta}$ the subset of firms of size $\beta$.

Similarly, $\mathcal{N}_{1}^{c}$ and $\mathcal{N}_{\beta}^{c}$ stand for the corresponding subsets of small and big firms within a component $c$. This leads to an effective size of component $c$ given by $K^{c}+1=\left|N_{1}^{c}\right|+\beta\left|N_{\beta}^{c}\right|$. In a component of this kind the expected fraction of firms who default (again in terms of their effective size), if indirectly hit by a shock $b$, is given by

$$
\begin{aligned}
& \frac{1}{K^{c}+1}\left[\sum_{i \in \mathcal{N}_{1}^{c}}\left(\sum_{j \in \mathcal{N}_{1}^{c} \backslash i} F\left(a_{i j}\right)+\beta \sum_{j \in \mathcal{N}_{\beta}^{c}} \operatorname{Pr}\left\{L>\frac{(R-M)}{a_{i j}}\right\}\right)+\right. \\
& \left.\sum_{i \in \mathcal{N}_{\beta}^{c}} \beta\left(\sum_{j \in \mathcal{N}_{1}^{c}} \operatorname{Pr}\left\{L>\frac{\beta(R-M)}{a_{i j}}\right\}+\beta \sum_{j \in N_{\beta}^{c} / i} \operatorname{Pr}\left\{L>\frac{\beta(R-M)}{a_{i j}}\right\}\right)\right]
\end{aligned}
$$

Let $V\left(K^{c},\left|\mathcal{N}_{1}^{c}\right|,\left|\mathcal{N}_{\beta}^{c}\right|\right)$ denote the value of the above expression when the pattern of linkages is chosen optimally, subject to constraints (10) (suitably adjusted to reflect the different effective sizes of the two types of firms) and (11). We show in the next proposition that in this case mixed components fare equally well, in terms of expected defaults, as homogeneous components. We have in fact:

Proposition 9 Under any of the conditions on the distribution of the b shocks stated in Propositions 2, 3, 5] or 6, for all $1 \leq K^{c} \leq N-1$ and all subsets $\mathcal{N}_{1}^{c} \subseteq \mathcal{N}_{1}$ and $\mathcal{N}_{\beta}^{c} \subseteq \mathcal{N}_{\beta}$ such that $\left|\mathcal{N}_{1}^{c}\right|+\beta\left|\mathcal{N}_{\beta}^{c}\right|=K^{c}+1$, we have $V\left(K^{c},\left|\mathcal{N}_{1}^{c}\right|,\left|\mathcal{N}_{\beta}^{c}\right|\right)=V\left(K^{c}\right)$.

Proof. Note that the expression in (34) of the expected fraction of firms defaulting in a component with $\left|\mathcal{N}_{1}^{c}\right|$ unit sized firms and $\left|\mathcal{N}_{\beta}^{c}\right|$ large firms can be conveniently rewritten as follows: 


$$
\frac{1}{K^{c}+1}\left[\sum_{i \in \mathcal{N}_{1}^{c}}\left(\sum_{j \in \mathcal{N}_{1}^{c} / i} F\left(a_{i j}\right)+\beta \sum_{j \in \mathcal{N}_{\beta}^{c}} F\left(a_{i j}\right)\right)+\sum_{i \in \mathcal{N}_{\beta}^{c}} \beta\left(\sum_{j \in \mathcal{N}_{1}^{c}} F\left(\frac{a_{i j}}{\beta}\right)+\beta \sum_{j \in \mathcal{N}_{\beta}^{c} / i} F\left(\frac{a_{i j}}{\beta}\right)\right)\right] .
$$

In addition, the balance condition $(12)$ is, in this case:

$$
\begin{aligned}
\sum_{j \in \mathcal{N}_{1}^{c} \backslash i} a_{i j}+\sum_{j \in \mathcal{N}_{\beta}^{c}} a_{i j} \beta & =1-\alpha, \quad \forall i \in \mathcal{N}_{1}^{c} \\
\sum_{j \in \mathcal{N}_{1}^{c} \backslash i} a_{i j} \frac{1}{\beta}+\sum_{j \in \mathcal{N}_{\beta}^{c} \backslash i} a_{i j} & =1-\alpha, \quad \forall i \in \mathcal{N}_{\beta}^{c} .
\end{aligned}
$$

Hence, if we proceed to the following change of variable $\widetilde{a}_{i j} \equiv a_{i j} / \beta$ for $i \in \mathcal{N}_{\beta}^{c}$ and all $j$ we see that the minimization problem whose solution yields $V\left(K^{c},\left|\mathcal{N}_{1}^{c}\right|,\left|\mathcal{N}_{\beta}^{c}\right|\right)$ becomes the same as problem $\left[\mathbf{P}\left(K^{c}\right)\right]$, subject to the additional constraint that at least $\beta$ terms in each row and column of $A_{K^{c}}$ take the same value. Note that the latter constraint is always satisfied at a solution of problem $\left[\mathbf{P}\left(K^{c}\right)\right]$ in all the cases considered in Section 2 (Propositions 2, 3, 5, 6), except when $F($.$) is$ uniformly concave) whose solution yields $V\left(K^{c}\right)$.

The proposition establishes an irrelevance result: the expected fraction of defaults in a component containing both large and unit sized firms is exactly the same as that in a component (of the same effective size) where all firms have unit size, provided in each of them the pattern of exposures is chosen optimally, subject to the constraints. The proof shows that, for any pattern of exposures $\left[\left(\bar{a}_{i j}\right)_{j \neq i}\right]_{i=1}^{K^{c}+1}$ in a homogeneous component of smaller firms, the same level of expected defaults can be attained in a mixed component, by appropriately rescaling the exposures of larger firms, setting $a_{i j}=\beta \bar{a}_{i j}$ for all $i \in \mathcal{N}_{\beta}^{c}$ and all $j$, while keeping unchanged the levels of exposures of smaller firms, $a_{k j}=\bar{a}_{k j}$ for all $k \in \mathcal{N}_{1}^{c}$ and all $j$.

The result can be understood in the light of the fact that we have constructed larger firms to be effectively the merger of $\beta$ smaller firms. It is interesting to note that an analogous result holds for other possible specifications of the effects of firms' size (for instance if $\beta$ affects the size of the shock, which is then given by $\beta l$, instead of the probability of being hit by a shock, or even a combination of the two). In this case the shock hitting a large firm is no longer the same as the 
one hitting $\beta$ small firms, but the equivalence result still holds, only the rescaling factors change.

We can say that Proposition 9 holds for reasons that are reminiscent of those behind the Modigliani-Miller theorem. The result shows that, as far as the effects on risk sharing and contagion are concerned, smaller firms are indifferent between forming linkages with firms of the same size or with larger firms. This stands clearly in contrast to the case where firms differ in terms of their risk characteristics.

\section{References}

[1] Acemoglu, D., A. Ozdaglar, and A. Tahbaz-Salehi. 2015. Systemic Risk and Stability in Financial Networks. American Economic Review 105:564-608.

[2] Acharya, V.V., L. H. Pedersen, T. Philippon and M. Richardson. 2017. Measuring Systemic Risk. Review of Financial Studies 30(1):2-47.

[3] Ahrend, R. and A. Goujard. 2011. Drivers of Systemic Banking Crises: The Role of BankBalance-Sheet Contagion and Financial Account Structure. OECD Working Paper Vol. 71, No. 902.

[4] Allen, F. and A Babus. 2009. Networks in Finance. In P. Kleindorfer and J. Wind (Eds.), The Network Challenge (367-382). Philadelphia, PA: Wharton School Publishing.

[5] Allen, F., A. Babus and E. Carletti. 2012. Asset Commonality, Debt Maturity and Systemic Risk. Journal of Financial Economics 104:519-534.

[6] Allen, F. and D. Gale. 2000. Financial Contagion. Journal of Political Economy 42:1-33.

[7] Alvarez, F. and G. Barlevy. 2014. Mandatory Disclosure and Financial Contagion. Federal Reserve Bank of Chicago Working Paper No. 2014-04.

[8] Ambrus A., M. Mobius, and A. Szeidl. 2014. Consumption Risk-sharing in Social Networks. American Economic Review 104(1):149-182

[9] Babus, A. 2016. The Formation of Financial Networks. RAND Journal of Economics 47:239272. 
[10] Battiston, S., M. Puliga, R. Kaushik, P. Tasca and G. Caldarelli. 2012. DebtRank: Too Central to Fail? Financial Networks, the FED and Systemic Risk. Scientific Reports 2:541.

[11] Beale, N., D. G. Rand, H. Battey, K. Croxson, R. M. May, and M. A. Nowak. 2011. Individual versus systemic risk and the Regulator's Dilemma. Proceedings of the National Academy of Sciences of the USA 108(31):12647-12652.

[12] Berglöf, E., and E. Perotti. 1994. The governance structure of the Japanese financial keiretsu. Journal of Financial Economics 36(2):259-284.

[13] Bloch, F., G. Genicot, and D. Ray. 2008. Informal Insurance in Social Networks. Journal of Economic Theory 143:36-58.

[14] Bramoullé, Y. and R. E. Kranton. 2007. Risk-Sharing Networks. Journal of Economic Behavior and Organization 64:275-94.

[15] Blume, L., D. Easley, J. Kleinberg, R. Kleinberg, É. Tardos. 2011. Network Formation in the Presence of Contagious Risk. Proc. 12th ACM Conference on Electronic Commerce.

[16] Caballero, R. and A. Simsek. 2013. Fire Sales in a Model of Complexity. The Journal of Finance 68:2549-2587.

[17] Cabrales, A., D. Gale and P. Gottardi. 2016. Financial Contagion in Networks. In Y. Bramoulle, A. Galeotti and B.W. Rogers (Eds.), The Oxford Handbook of Network Economics. Oxford: OUP.

[18] Cabrales, A., P. Gottardi, and F. Vega-Redondo. Risk-Sharing and Contagion in Networks. CESifo Working Paper Series No. 4715.

[19] Calvó-Armengol, A. and R. Ikiliç. 2009. Pairwise Stability and Nash Equilibria in Network Formation. International Journal of Game Theory 38:51-79.

[20] Degryse, H. and G. Nguyen. 2007. Interbank Exposures: an Empirical Examination of Systemic Risk in the Belgian Banking System. International Journal of Central Banking 3(2):123-171.

[21] Denbee, E., C. Julliard, Y. Li and K. Yuan. 2011. Network Risk and Key Players: A Structural Analysis of Interbank Liquidity. Working paper. 
[22] Dewenter, K. L. 2003. The risk-sharing role of Japanese keiretsu business groups: evidence from restructuring in the 1990s. Japan and the World Economy 15(3):261-274.

[23] Diebold, F.X. and K.Yilmaz. 2009. Measuring Financial Asset Return and Volatility Spillovers, with Application to Global Equity Markets. Economic Journal 119:158-171.

[24] DTCC (Depository Trust \& Clearing Corporation). 2015. Understanding Interconnectedness To Build A More Resilient Financial System. DTCC White papers.

[25] Elliott, M., B. Golub and M. O. Jackson. 2014. Financial Networks and Contagion. American Economic Review, 104: 3115-3153.

[26] Elsinger, H., A. Lehar and M. Summer. 2006. Risk Assessment for Banking Systems. Management Science 52(9):1301-1314 .

[27] Gai, P., A. Haldane, and S. Kapadia. 2011. Complexity, Concentration and Contagion. Journal of Monetary Economics 58:453-470.

[28] Glasserman, P., and H. P. Young. 2015. How Likely is Contagion in Financial Networks? Journal of Banking and Finance 50:383-399.

[29] Goyal, S. and F. Vega-Redondo. 2007. Structural holes in social networks. Journal of Economic Theory 137:460-492.

[30] Greif, A., and C. Kingston. 2011. Institutions: Rules or Equilibria? In G. Caballero et al. (Eds.), Political economy of institutions, democracy and voting (pp. 13-43). Berlin: Springer.

[31] Kroszner, R.S. and R.G. Rajan. 1994. Is the Glass-Steagall Act Justified? A Study of the U.S. Experience with Universal Banking Before 1933. The American Economic Review 84: 810-832.

[32] Jackson, M. and A. Wolinsky. 1996. A Strategic Model of Social and Economic Networks. Journal of Economic Theory 71:44-74.

[33] Khanna, T., and Y. Yafeh. 2005. Business groups and risk sharing around the world. The Journal of Busines 78(1):301-340. 
[34] Krugman, P. 1999. Balance Sheets, the Transfer Problem, and Financial Crises. International Tax and Public Finance 6:459-472.

[35] Lazear, E. P. 2011. The Euro Crisis: Doubting the 'Domino' Effect. The Wall Street Journal, October 31, 2011, http://online.wsj.com/articles/SB10001424052970203554104577003924075089102

[36] Leitner, Y. 2005. Financial Networks: Contagion, Commitment and Private Sector Bailouts. Journal of Finance 60:2925-2953.

[37] Loepfe, L., A. Cabrales and A. Sánchez. 2013. Towards a proper assignment of systemic risk: the combined roles of network topology and shock characteristics. PLoS ONE 8.

[38] Myerson, R. 1991. Game Theory: Analysis of Conflict. Cambridge, MA: Harvard University Press.

[39] Nier, E., J. Yang, T. Yorulmazer and A. Alentorn. 2007. Network Models and Financial Stability. Journal of Economic Dynamics and Control 31:2033-2060.

[40] Osano, H. 1996. Intercorporate shareholdings and corporate control in the Japanese firm. Journal of Banking \& Finance 20(6):1047-1068.

[41] Stiglitz, J.E. 2010. Risk and Global Economic Architecture: Why Full Financial Integration May Be Undesirable. American Economic Review: Papers $\&$ Proceedings 100:388-392. 